\title{
Neue Herausforderungen erfordern neue Ideen: Elemente einer Theorie des Sieges in modernen strategischen Konflikten
}

https://doi.org/10.1515/sirius-2020-4004

Zusammenfassung: In Russland und China dreht sich der militärstrategische Diskurs schon seit Langem um die Frage, wie man die Vereinigten Staaten in einem regionalen konventionellen Krieg unter dem nuklearen Schatten abschrecken und besiegen kann. Bis vor Kurzem lag das Hauptaugenmerk des militärstrategischen Denkens der USA auf Terrorismus- und Aufstandsbekämpfung. Dies hat zu einem gefährlichen Ungleichgewicht und zu dem Risiko geführt, dass die Führungen in Moskau und Peking versucht sein könnten, in Konflikte einzutreten und diese zu eskalieren, in der Überzeugung, sie könnten diese gewinnen. Die USA und ihre Verbündeten müssen daher eigene Konzepte darüber entwickeln, wie sie diese Überzeugung erschüttern und folglich die Abschreckung stärken können. Eine kohärente, glaubwürdige Gesamtheit diesbezüglicher Ideen könnte auch eine „Theorie des Sieges“ genannt werden.

Schlüsselbegriffe: Strategie, Theorie des Krieges, Konfliktmanagement, strategische Rivalität, Russland, China

Abstract: In Russia and China, military thinking has long focused on the problem of deterring and defeating the US in a regional conventional war under the nuclear shadow. Until recently, the military thinking of the US has focused on counter-terrorism and counter-insurgency. The result is a dangerous imbalance and a risk that leaders in Moscow

Anmerkung: Dieser Aufsatz ist ein Auszug aus einer längeren Monografie mit dem Titel Theories of Victory, Red and Blue, die als Paper No. 7 in der CGSR Livermore Paper Series veröffentlicht wurde. Die hier geäußerten Ansichten sind die persönliche Meinung des Autors und sollten nicht seinem Arbeitgeber oder seinen Förderern zugeschrieben werden.

*Kontakt: Dr. Brad Roberts ist Direktor des Center for Global Security Research am Lawrence Livermore National Laboratory in Livermore, California. Von 2009 bis 2013 war er U.S. Deputy Assistant Secretary of Defense für Nuklear- und Raketenabwehrstrategie. E-Mail: roberts86@llnl.gov and Beijing might be tempted to enter conflict and escalate, confident they can win. The US and its allies must develop ideas of their own about how to strip away such confidence and thus strengthen deterrence. A coherent, credible collection of ideas can be thought of as a theory of victory.

Keywords: Strategy, theory of war, management of conflicts, strategic rivalry, Russia, China

\section{Einleitung}

Die Vereinigten Staaten könnten den nächsten großen Krieg durchaus verlieren - nicht, weil es ihnen an den richtigen Fähigkeiten mangelte, sondern, weil sie nicht die mühsame intellektuelle Arbeit geleistet haben, die grundlegenden Merkmale regionaler konventioneller Kriege zu verstehen, bei denen ihre Gegner zu einer domänenübergreifenden, transregionalen Eskalation fähig sind. Dies war die zentrale Schlussfolgerung der parteiübergreifenden National Defense Strategy Commission (NDSC) in ihrem im November 2018 veröffentlichten Bericht. ${ }^{1}$

Im etablierten Washingtoner Politikbetrieb gibt es ein reges Kommen und Gehen von Kommissionen. Ihr Einfluss auf die Politik ist in der Regel von kurzer Dauer, weil Interessenvertreter sich anderen Dingen zuwenden und neue Fragen auftauchen. Aber dieser Bericht verdient größere Beachtung und verlangt nach einer fundierten Antwort. Wer glaubt, die Kritik komme nur von einer verschrobenen Kommission, der sollte bedenken, zu welcher Einschätzung General Joseph Dunford gelangte, der als Vorsitzender der Vereinigten Stabschefs im Jahr 2016 erklärte, dass „wir schon jetzt in vielerlei Hinsicht bei der Anpassung

1 Edelman/Roughead/Fox/Hicks/Keane/Krepinevich/Kyl/Mahnken/McCord/Morell/Patterson/Zakheim 2018. Die beiden Vorsitzenden der Kommission waren der ehemalige Staatssekretär (für Strategie) im US-Verteidigungsministerium Eric Edelman und der ehemalige Admiralstabschef der US Navy Gary Roughhead. 
an den veränderten Charakter der heutige Kriegsführung im Hintertreffen sind.“2 Man sollte auch die Ansicht des Direktors der militärwissenschaftlichen Abteilung am Royal United Services Institute in London, Peter Roberts, einbeziehen, der 2017 schrieb, dass ,potentielle Gegner ... neue Konzepte der Kriegsführung und neue Sichtweisen auf Konflikte entwickelt haben, bei denen sie sich nicht die Beschränkungen auferlegen, die westlichen Denkgewohnheiten zu eigen sind ... Der Glaube an die Überlegenheit westlicher Konzepte und Ideen ist nach wie vor tief im herrschenden westlichen Diskurs verwurzelt; diese Überheblichkeit birgt erhebliche Gefahren." ${ }^{3}$

Die Vereinigten Staaten sind schon jetzt „im Hintertreffen," weil Russland und China schon seit dreißig Jahren daran arbeiten, Ordnung in ihre Theoriegebäude zu bringen. Sie haben verlässlich und kontinuierlich neue strategische Konzepte entwickelt, die uns beunruhigen sollten. Russische und chinesische Planer haben „neue Konzepte der Kriegsführung und neue Sichtweisen auf Konflikte“ mit den Vereinigten Staaten entwickelt, die wir erst allmählich verstehen. Sie studierten die amerikanische Kriegsführung unter anderem in Kuwait, Irak, Kosovo und in Afghanistan. Sie haben die regelmäßigen Überprüfungen der US-Verteidigungspolitik, der US-Militärstrategie und der militärischen Fähigkeiten genau verfolgt und analysiert, um Rückschlüsse auf die militärischen Ziele der USA und die zukünftige amerikanische Kriegsführung zu ziehen. Anschließend überarbeiteten sie ihre Militärstrategien, entwickelten neue Einsatzkonzepte, passten ihre militärischen Organisationsstrukturen an, entwickelten und testeten neue Doktrinen und entwarfen, erwarben und führten neue Fähigkeiten ein, die mit diesen Konzepten und Doktrinen in Einklang standen. Dann brachten sie den politischen Willen und die nachhaltige Fokussierung auf, um die erheblichen bürokratischen, technischen und finanziellen Hindernisse bei der Umsetzung der neuen Theorien zu überwinden.

Ihre intellektuellen Anstrengungen konzentrierten sich auf ein Gebiet, das nach dem Dafürhalten der meisten amerikanischen Militärexperten von den USA dominiert wird: der Bereich eskalierender Kampfmaßnahmen jenseits des Einsatzes regulärer konventioneller Streitkräfte. Aber da, wo wir unsere Stärken verorten, erkannten Experten in Russland und China Gelegenheiten. Diese Inkongruenz ist nach Ansicht der NDSC besonders beunruhigend: Gegner der USA haben neue Konzepte dafür entwickelt,

2 Rede auf der Graduiertenfeier der National Defense University, Ft. McNair, D.C., 10. Juni 2016.

3 Roberts 2017. wie sich solche Konflikte dadurch steuern lassen, dass man die Entscheidungen seiner Gegner in einer Weise beeinflusst, die den eigenen nationalen Zielen förderlich ist. Ziel ist es, seinen Gegner durch Eskalation und Androhung des massiven Einsatzes weiterer Waffen Kosten und Risiken aufzuerlegen.

Diese Ideen ergeben in ihrer Gesamtheit das, was man durchaus eine Theorie des Sieges nennen kann. ${ }^{4}$ Da dieser Begriff nicht allgemein gebräuchlich ist, müssen wir ihn definieren. Was ist eine Theorie des Sieges? Beginnen wir mit dem, was sie nicht ist: Sie ist keine Strategie. Nach Thomas Schellings grundlegender Begriffsbestimmung ist eine Strategie eine „rationale, zielgerichtete und ausgeklügelte Form von Verhalten, die darauf abzielt, einen Wettstreit zu ,gewinnen'. " ${ }^{5}$ Eine Strategie sollte uns sagen, was wir tun sollen und wie wir es tun sollen. Eine erfolgversprechende Strategie sollte Handlungen auf plausible Weise mit angestrebten Ergebnissen verknüpfen. In den Handbüchern der Militärakademien versteht man unter Strategie eine Vorgehensweise, die Ziele, Methoden und Mittel aufeinander abstimmt. Es erscheint naheliegend, dass eine Strategie für „das Gewinnen eines Wettstreits“ eine Theorie über den Weg dorthin - also den Sieg - enthalten sollte. Aber über die Logik hinter den Verknüpfungen von Handlungen und Ergebnissen macht eine Strategie nicht unbedingt genauere Angaben. In dem Konstrukt aus Zielen, Methoden und Mitteln bleibt die Theorie unausgesprochen, auch wenn die Ziele, Methoden und Mittel aufeinander abgestimmt sind. Sie ist implizit, nicht explizit.

Folglich besteht eine Theorie des Sieges aus einer Reihe von Aussagen darüber, wie und warum das Verhalten eines Teilnehmers an einem Krieg oder an einem Konflikt unterhalb der Schwelle zum Krieg das Verhalten eines anderen Kriegs-/Konfliktteilnehmers in der gewünschten Weise beeinflussen könnte. Diese Theorie ist ein durchgehender Faden, der sich mit einer „,inneren Logik“ und mit „kausalen Verknüpfungen“ zwischen Zielen, Methoden und Mitteln durch jede Strategie zieht. ${ }^{6}$ Bezugnehmend auf Clausewitz, könnte man sagen, dass eine Theorie des Sieges erklärt, wie man einen Feind an einen „Kulminationspunkt" bringt, an dem er beschließt, die Kosten und Risiken einer Fortsetzung des Konfliktes nicht zu tragen und sich stattdessen den Präferenzen des stärkeren Akteurs zu fügen, indem er den Konflikt beendet. Eine Variante beruft sich auf Sun Tzu - Sieg bedeutet hier kampfloses Bezwingen eines Feindes.

\footnotetext{
4 Roberts 2015. 5 Schelling 1960a, 3. 6 Hoffman 2020.
} 
Es gibt eine „rote Theorie“ des Sieges. Das ist eine Gesamtheit von Ideen, die von Gegnern der Vereinigten Staaten entwickelt wurden und die sich um die Frage drehen, wie man die Vereinigten Staaten und ihre Verbündeten dazu bringen könnte, eine von den Gegnern angestrebte regionale Ordnung anzuerkennen und, falls nötig, wie man die USA vom militärischen Eingreifen abschrecken und ihnen in Krisen und Kriegen eine Niederlage beibringen könnte. Diese rote Theorie des Sieges basiert auf folgenden Annahmen: Erstens, auf der Basis eines militärischen fait accompli (z. B. einer überraschenden Landnahme oder eines schnellen Staatsstreiches) soll eine entschlossene militärische Antwort der Vereinigten Staaten dadurch abgewendet werden, dass man Interessenunterschiede und Spaltungen zwischen den USA und ihren Verbündeten ausnutzt. Zweitens, die Vereinigten Staaten sollen dazu gebracht werden, ein wichtiges regionales Interesse aufzugeben, anstatt ihr gesamtes militärischen Potenzial einzusetzen. Es gilt, die USA davon zu überzeugen, dass ihr Interesse nicht so bedeutend ist, dass sie sich dafür langfristig auf eine Strategie der Drohung mit dem Äußersten (brinkmanship) und der kompetitiven Eskalation einlassen würden. Das rote Konzept des Sieges beinhaltet mehr als nur das Erlangen und Festhalten eines Vorteils. Es erstreckt sich auch auf die Entscheidung von „Blau“, den Konflikt zu Bedingungen zu beenden, unter denen es das Interesse opfert, welches es bislang verteidigt hat. Damit soll aufgezeigt werden, dass auf die regionalen Sicherheitsordnungen der USA sowie auf deren Rolle als Garantiemacht kein Verlass ist. Das Konzept beinhaltet auch Bemühungen, die Vereinigten Staaten und ihre Verbündeten dazu zu bewegen, ihre Interessen nicht in Konfrontationen knapp unterhalb der Schwelle bewaffneter Feindseligkeiten zu verteidigen (das, was heute „Grauzonen-Konflikt" genannt wird).

Es gibt derzeit keine vergleichbare „blaue“ Theorie des Sieges. Die Vereinigten Staaten und ihre Verbündeten haben einige Elemente einer Theorie, aber kein kohärentes Ganzes. Bis ungefähr 2014 waren wir zu sehr damit beschäftigt, uns über Worte zu streiten, und wir waren allzu beeindruckt von unserer militärischen Überlegenheit, als dass wir diese Aufgabe ernst genommen hätten. Nach 2014 haben wir den Preis von dreißig Jahren strategischer Verkümmerung in Form eines Wirrwarrs alter Konzepte, der Verlockung schneller Verlegenheitslösungen und begrenzter analytischer Werkzeuge für neue Probleme bezahlt.

Eine blaue Theorie des Sieges ließe sich in einem dreistufigen Prozess ausarbeiten: Erstens, man geht bei Rot in die Schule, so wie Rot bei Blau in die Schule gegangen ist. Zweitens, man entwickelt ein allgemein gehaltenes Gegenmodell zu der roten Rahmentheorie des Sieges. Drittens, man schneidet dieses Modell auf spezifische regionale Kontexte zu. Dabei muss das gesamte Konfliktspektrum abgedeckt werden: von Konfrontationen unterhalb der Schwelle bewaffneter Feindseligkeiten über Krisen bis hin zu Kriegen. Ihr Kernkonzept sollte nicht Abschreckung oder Eskalationskontrolle sein; vielmehr sollte sie sich darauf konzentrieren, das Vertrauen der Führungsverantwortlichen in Russland und China in ihr Eskalationskalkül zu erschüttern. Dieses besteht in ihrer Einschätzung der Vorteile, Kosten und Risiken von Eskalationsschritten in Krisen und Kriegen und auch in der Grauzone. Blau muss in der Lage sein, der roten Seite den von ihr erwarteten Nutzen aus Handlungen zu verringern, die unter der roten Theorie des Sieges durchgeführt wurden. Zugleich muss sie die erwarteten Kosten und Risiken für Rot erhöhen. Man könnte dies als eine Gegeneskalationsstrategie - im Unterschied zu einer Eskalationsdominanzstrategie - bezeichnen. Die blaue Theorie des Sieges muss auch die Abschreckungserfordernisse in einem zweiten Einsatzgebiet berücksichtigen, aus dem in Krisen- oder Kriegszeiten womöglich Ressourcen abgezogen werden (Europa oder Ostasien). Eine glaubhafte Theorie des Sieges in dem zweiten, dann nicht ausreichend ausgestatteten Einsatzgebiet erfordert es, dass sich die Vereinigten Staaten stärker auf Verteidigungs- und Abschreckungsfähigkeiten ihrer Alliierten stützen und darüber hinaus auch eine größere Bereitschaft zeigen, nukleare Abschreckung für dieses Gebiet ins Spiel zu bringen.

\section{Theorien des militärischen Sieges von Russland und China}

Während die militärischen Planer in Washington nach dem Ende des Kalten Krieges weitgehend aufhörten, sich den Kopf über Russland und China zu zerbrechen, haben militärische Planer in Moskau und Peking nicht aufgehört, über die Vereinigten Staaten nachzudenken. Die Schnelligkeit, mit der die Vereinigten Staaten und ihre Koalitionspartner das irakische Militär außer Gefecht setzten, sowie die Effektivität, mit der die USA eine bunt gemischte internationale Koalition zusammenstellten und anführten, ließ in beiden Hauptstädten die Alarmglocken schrillen. Das Interesse daran, wie souverän die Vereinigten Staaten die sogenannte Revolution im Militärwesen meisterten, wuchs noch durch den Kosovo-Krieg und dadurch, dass es der NATO gelang, ohne Entsendung von Bodentruppen die Dinge nachhaltig zu wenden und 
sich durchzusetzen. Die Entsendung von zwei Flugzeugträgerkampfgruppen in die Straße von Taiwan im Jahr 1995 und die anschließende Bombardierung der chinesischen Botschaft in Belgrad motivierten die chinesischen Militärplaner zusätzlich; schienen die USA Peking doch $\mathrm{zu}$ signalisieren, dass sie gewillt und bereit für einen Krieg mit China seien.

Aber die 1990er-Jahre waren für diese Länder in wirtschaftlicher und politischer Hinsicht eine schwierige Zeit, und keines von beiden verfügte über die Ressourcen für zeitnahe militärische Lösungen der Unipolarität. Die russische Wirtschaft war im Begriff zusammenzubrechen, und der chinesische Boom stand noch ganz am Anfang. Anstatt das Problem mit Geld, neuen Technologien oder Truppenstrukturreformen anzugehen, setzten beide Regierungen auf Menschen. Jedes Land investierte in das Humankapital und die institutionelle Infrastruktur, um die benötigten militärischen und strategischen Konzepte für die neue Ära zu entwickeln. ${ }^{7}$ Was genau taten sie dabei? Der stellvertretende US-Verteidigungsminister Robert Work erklärte im Jahr 2016: „Während die Vereinigten Staaten und unsere engsten Verbündeten in den letzten 13 Jahren zwei lang andauernde Kriege führten, sahen der Rest der Welt und unsere potentiellen Gegner, wie wir operierten. Sie schauten sich unsere Stärken an ... sie hielten Ausschau nach unseren Schwächen. Und dann begannen sie, Mittel und Wege zu ersinnen, um unserer technologischen Überlegenheit entgegenzutreten." ${ }^{* 8}$

Anscheinend erkundeten sie das gesamte Spektrum potenzieller Auslöser von Konflikten mit den Vereinigten Staaten und deren Verbündeten, die amerikanische Strategie der Kriegsführung (insbesondere ihre Stärken und Schwächen), die Pläne und Ambitionen der USA, das Potenzial der fortgeschrittenen konventionellen Kriegsführung (und der neuen Domänen) und die Struktur und die Eigenarten regionaler/lokaler Kriege. Was lernten sie dadurch, dass sie bei der amerikanischen Kriegsführung in die Schule gingen? Wir wissen es nicht mit Sicherheit, aber es gibt einige aufschlussreiche Indizien. ${ }^{9}$ So können wir uns eine ungefähre Vorstellung von dem machen, was sie gelernt haben könnten:

- Das US-Militär kann seine konventionelle Überlegenheit nur in einem lang andauernden Krieg oder

7 Mulvenon/Finkelstein 2005.

8 Stellvertretender Verteidigungsminister Robert Work: The Third U.S. Offset Strategy and Its Implications for Partners and Allies. Washington, D.C., 28 Januar 2015.

9 Diese Zusammenfassung stützt sich auf Roberts 2015. Vgl. auch Thomas 2015 und Peng/Yao 2015. in einem Krieg mit langem Vorlauf ausspielen. Aber diese Überlegenheit beginnt auch zu erodieren.

- Um in einem konventionellen Konflikt die Oberhand zu gewinnen, müssen die Vereinigten Staaten Truppen und Material über große Entfernungen verlegen und von Basen, Häfen und Flughäfen aus operieren, die ein leichtes Ziel für Angriffe und Störmanöver sind.

- Bei der Verlegung von Truppen und Material sind die Vereinigten Staaten in hohem Maße von Verbündeten abhängig - auf operativer und politischer Ebene. Sie könnten allein handeln, entscheiden sich jedoch möglicherweise dagegen.

- Die Vereinigten Staaten haben sich zum Ziel gesetzt, dominante Positionen im Cyberspace und im Weltraum zu besetzen und zu halten und so das Nutzenpotenzial der Revolution im Militärwesen vollständig auszuschöpfen. Aber in diesen Domänen sind sie auch verwundbar.

- Sie streben außerdem an, sich die Innovationsstärke ihrer Privatwirtschaft zunutze zu machen, um sich mit neuen Technologien wie etwa Hyperschall-Antrieben und Künstlicher Intelligenz (KI) mögliche strategische Vorteile zu verschaffen.

- Die strategischen Atomstreitkräfte der USA sind weiterhin zu glaubhafter Abschreckung fähig und effektiv, aber die für die erweiterte Abschreckung eingesetzten nuklearen Einsatzmittel wurden nach dem Ende des Kalten Krieges drastisch reduziert. Das, was übriggeblieben ist, ist weitgehend veraltet.

- Die amerikanische Öffentlichkeit ist skeptisch gegenüber Kriegen, toleriert kaum eigene Verluste an Menschenleben und wünscht sich schnelle, entscheidende und unblutige Siege. Sie setzt die politische Klasse entsprechend unter Druck.

Militärexperten in Russland und China haben nach der eingehenden Analyse der amerikanischen Kriegsführung neue Strategien und Einsatzkonzepte sowie dafür maßgeschneiderte Waffenkonzepte entwickelt. Nachdem sie ihre intellektuelle Arbeit abgeschlossen hatten, nahmen sie die notwendigen Reformen von Institutionen und Prozessen in Angriff. Diese Veränderungen begannen im Jahr 1993 mit Überarbeitungen der nationalen Militärstrategie in beiden Ländern, die den Schwerpunkt auf regionale Kriege an ihren Peripherien gegen einen Hightech-Gegner verlagerten. Seither hat Russland seine Militärstrategie mehrfach - in den Jahren 2000, 2010 und 2014 - grundlegend überarbeitet, während China jährlichen Weißbüchern zur Verteidigungspolitik ein besonderes Gewicht einräumt. Und wohl in dem Bestreben, ausländische Experten mit ihren Fortschritten - als eine Form der Abschreckungssi- 


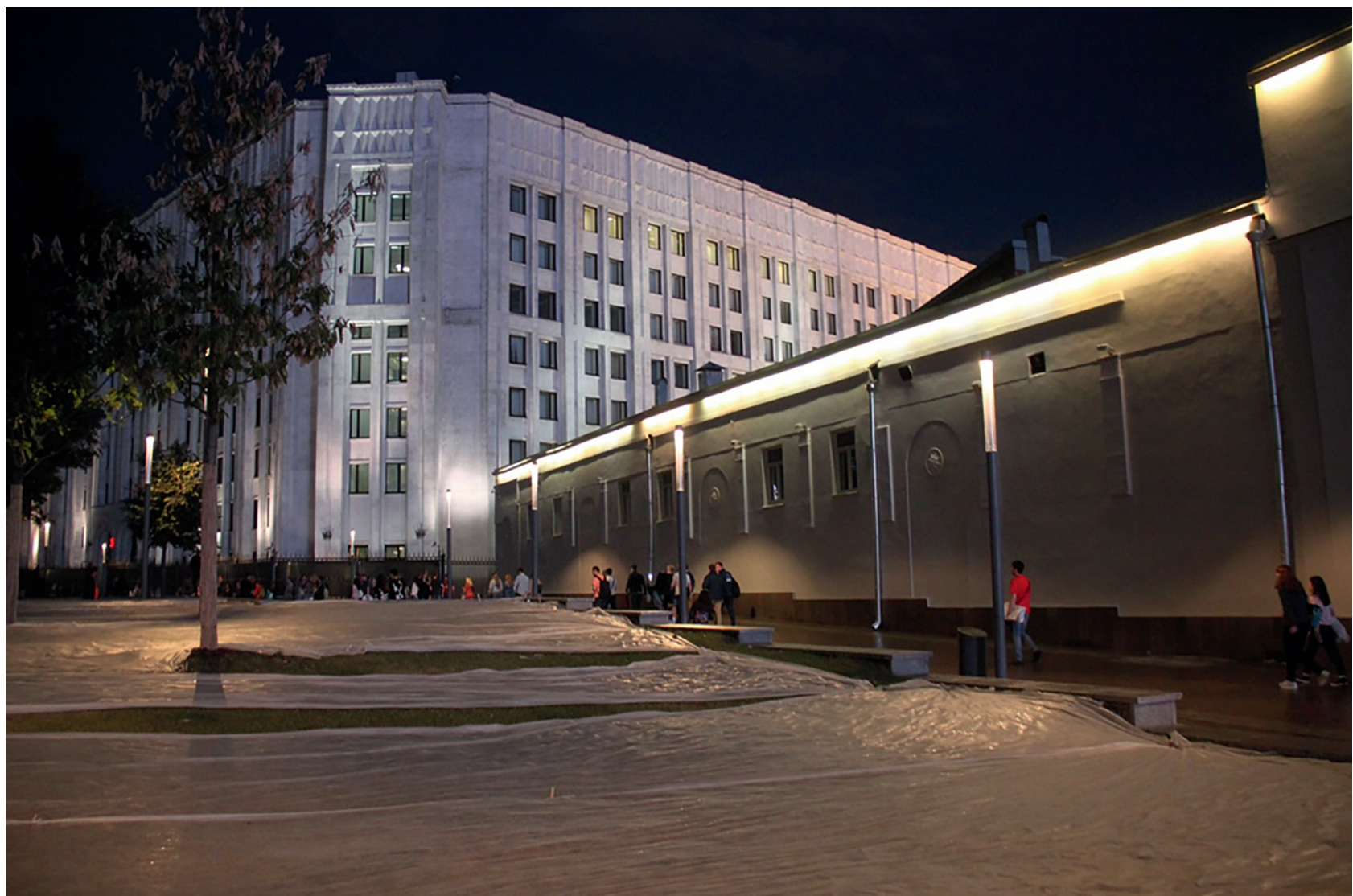

Abb 1: Gebäude des russischen Generalstabs in Moskau

gnalisierung - zu beeindrucken, machten sie einen Großteil dieser neuen Konzepte Außenstehenden zugänglich: ob in Fachpublikationen (Russland) oder in Unterrichtsmaterialien (China).

Selbstverständlich war und sind die militärischen Herausforderungen, welche die Vereinigten Staaten für China und Russland darstellen, nicht das einzige Problem, mit dem die beiden Länder konfrontiert sind. China schlägt sich zudem mit ungeklärten Grenzstreitigkeiten mit Indien und mit Defiziten bei seinen Fähigkeiten zur maritimen Machtprojektion herum. Russland sieht sich außerdem dem Problem lokaler Kriege (z. B. Tschetschenien) und Schwächen bei der Machtprojektion jenseits seiner unmittelbaren Peripherie (z.B. in den Nahen Osten) gegenüber. Oscar Jonsson warnte davor, Russland eine monolithische Sicht moderner Kriegsführung zuzuschreiben. Im Jahr 2019 schrieb er: „Es gibt weder in Russland noch irgendwo sonst eine einheitliche Theorie des Krieges. Vielmehr gibt es eine Gesamtheit verschiedener Sichtweisen, die mehr oder minder miteinander übereinstimmen können. Dennoch ermöglicht die Analyse der Konzeptionen des Krieges auf diesen ... verschiedenen Gebieten, dass man sich eine näherungsweise Vorstel- lung von der Kriegsauffassung und ihrer Entstehungsgeschichte machen kann." ${ }^{10}$

\subsection{Schlüsselparameter der neuen strategischen Herausforderung}

Die Wahrnehmungen, Beschlüsse und Strategien wichtiger Entscheidungsträger in Moskau und Peking haben für die Vereinigten Staaten und den Westen im Allgemeinen eine neue strategische Herausforderung geschaffen ein Problem, das eine umfassende und langfristige politisch-militärische Reaktion erfordert, die maßgeblich von einer Theorie des Sieges beeinflusst wird. Diese neue Herausforderung zeichnet sich durch die folgenden grundlegenden Rahmenbedingungen aus:

- Aus russischer und chinesischer Sicht ist der Konflikt, in dem sie sich gegenwärtig mit den USA und dem Westen befinden, ein lang anhaltender und beständiger. Gegenwärtig und aufgrund ihrer strategischen

10 Jonsson 2019, 17. 
Kultur vielleicht dauerhaft, teilen sie nicht die feinsäuberliche Unterscheidung zwischen Krieg und Frieden, die in den USA üblich ist. Die Regierenden in Moskau und Peking sehen sich bereits im Krieg mit den Vereinigten Staaten und dem Westen - einem Krieg, der heute mit Mitteln der Soft Power auf einer Hard-Power-Grundlage ausgetragen wird. Moskau und Peking konkurrieren (mit den USA) mit Mitteln, die knapp unterhalb der Schwelle eines Krieges liegen, um das Gleichgewicht von Macht und Einfluss zu verschieben. Sie stellen die Fähigkeiten zusammen und schaffen die Bedingungen, unter denen sie ihre Führungsambitionen verwirklichen können. Sie setzen militärische Mittel bei Operationen knapp unterhalb der Kriegsschwelle ein, um neue geopolitische Ansprüche zu erheben und zu verteidigen und um allmählich regionale Ordnungen und die Glaubwürdigkeit der USA zu untergraben. Der Generalstabschef der russischen Streitkräfte, General Valeri Gerassimov, drückte es folgendermaßen aus: „Heute ist es offensichtlich, dass die Grenze zwischen Krieg und Frieden verschwimmt." ${ }^{11}$ Dies hat eine wichtige Konsequenz für die blaue Theorie des Sieges. Sie muss nämlich sowohl bewaffnete Feindseligkeiten (und die ihnen vorausgehenden politisch-militärischen Krisen) als auch Konflikte der Grauzone (und die regelmäßig wiederkehrenden Krisen, die mit ihr verbunden sind) abdecken.

- Russland und China haben sich auf regionale Kriege vorbereitet. Hier geht es um von den USA unterstützte regionale Ordnungen, die durch regionale Kriege zerstört werden können. Es geht aber auch um die Abwehr angeblicher Versuche der USA, autoritäre Regime durch sogenannte „farbige Revolutionen“ zu Fall zu bringen. Regionale Kriege sind keine totalen Kriege. Diese neue Herausforderung ist nicht der totale Krieg, wie er in der ersten Hälfte des 20. Jahrhunderts konzipiert wurde, oder ein atomarer Krieg, wie man sich ihn im Kalten Krieg vorstellte, oder ein „uneingeschränkter Krieg,“ über den einige chinesische und andere Experten in den ersten Jahren nach dem Kalten Krieg spekulierten. ${ }^{12}$ Der vorrangige militärische Fokus Chinas liegt auf „lokal (begrenzten) Kriegen unter Bedingungen von Hightech und Informationsdominanz." ${ }^{13}$ Der vorrangige militärische Fokus Russlands

11 Valery Gerasimov: World on the Brink of War, Military-Industrial Courier, (15. März 2017); https://vpk-news.ru/articles/35591.

12 Qiao/Wang 1999.

13 Information Office of the State Council: White Paper on National Defense. Beijing 2015; http://www.china.org.cn/china/2015-05/26/ content_35661433.htm. liegt auf einem regionalen Krieg in Europa (unter Beteiligung der Vereinigten Staaten). Hier kommt es darauf an, dass regionale Kriege gewonnen und dann begrenzt werden. Russland wie China bereiten sich darauf vor, sich gegen Bedrohungen ihrer Interessen durch die USA an ihrer Peripherie zu verteidigen und die durch die USA gestalteten regionalen Sicherheitsordnungen in ihrem Umfeld herauszufordern und zu zerstören.

Diese beiden Rahmenbedingungen haben wichtige Konsequenzen für die blaue Theorie des Sieges: Diese muss sowohl den spezifischen regionalen Kontext, in dem Russland und China vorgehen, berücksichtigen wie auch die maßgeblichen Unterschiede zwischen einem eingeschränkten und einem uneingeschränkten Krieg zwischen Großmächten. In solchen regionalen Kriegen wären US-Verbündete die Hauptziele, und der Hauptgewinn wäre ihre politische „Gleichschaltung.“ Die Vereinigten Staaten wären weder das Hauptziel noch der Hauptgewinn. Da es Moskau, Peking oder Pjöngjang in solchen Kriegen vor allem darum ginge, die regionalen Ordnungen umzugestalten, müsste ihr Ziel darin bestehen, die Beziehungen zwischen den Vereinigten Staaten und ihren Verbündeten zu unterminieren. Sie wollten dies vermutlich dadurch erreichen, dass sie den Alliierten ihre Schwächen und die praktischen Schwierigkeiten für die Vereinigten Staaten, sie zu beschützen, in Erinnerungen riefen und Zweifel an der Glaubwürdigkeit der USA weckten.

Daraus ergibt sich eine weitere wichtige Konsequenz für die blaue Theorie des Sieges. Diese muss nämlich die Interessen und Anliegen der US-Verbündeten berücksichtigen. Sie muss auch deren potenzielle Beiträge zur gemeinsamen Verteidigung, ihre eigenen Theorien des Sieges und ihre Fähigkeit zu Vorgehensweisen berücksichtigen, die unter Umständen von Washington nicht präferiert werden. Ungeachtet ihres regionalen und eingeschränkten Charakters hätten solche Kriege ein erhebliches Eskalationspotenzial. Eine Eskalation könnte auf vielfältige Weise stattfinden. Anhaltende Feinseligkeiten im Cyberspace und/oder im Weltraum könnten sich zu einem Zusammenstoß zwischen regulären Streitkräften ausweiten. Reguläre Streitkräfte, die bereits in Kämpfe verwickelt sind, könnten neue Einsatzregeln (rules of engagement) erhalten, die die Schwelle für die Anwendung tödlicher Gewalt absenkten, und/oder sie könnten Kampfmittel mit höherer Zerstörungskraft gegen ein breiteres Spektrum von Zielen anwenden. Angriffe niedriger Letalität könnten zu Angriffen hoher Letalität werden. Militärische Aktivitäten könnten auf zusätzliche Fronten und/oder auf zusätzliche Partner der Vereinigten Staaten 
ausgeweitet werden. Dies wird in der Nationalen Militärstrategie Chinas zum Ausdruck gebracht: „Die weltweite Revolution im Militärwesen erreicht ein neues Stadium. Weitreichende, präzise, intelligente, getarnte und unbemannte Waffen werden immer leistungsfähiger. Der Weltraum und der Cyberspace sind zu den neuen Kommandohöhen im strategischen Wettstreit zwischen allen Parteien geworden. Die Form der Kriegsführung beschleunigt ihre Entwicklung zur Informatisierung." ${ }^{\text {14 }}$

Angriffe auf US-Überseegebiete und die kontinentalen USA könnten ebenfalls - kinetisch und nicht kinetisch mit unterschiedlicher Letalität durchgeführt werden. Im Idealfall nähme man an, dass diese im Spätstadium eines Konflikts durchgeführt würden, in dem Bemühen, die Vereinigten Staaten dazu zu bringen, klein beizugeben, ehe sie erhebliche neue Kosten auf sich nähmen. Aber sie könnten auch frühzeitig in einem Konflikt stattfinden, um die Verlegung konventioneller US-Streitkräfte zu stören und vielleicht auch um zu versuchen, den politischen Willen Amerikas zu brechen.

Auch mit nuklearen Mitteln könnte Rot eine Eskalation vorantragen. Theoretisch könnten Kernwaffen gegen US- und alliierte militärische Ressourcen, gegen Hauptstädte von Verbündeten und andere Ziele und/oder gegen das US-Territorium eingesetzt werden. All diese Einsätze scheinen sehr unwahrscheinlich $\mathrm{zu}$ sein. Allerdings bedeutet das nicht, dass derartige Schläge unmöglich oder unplausibel sind. Es gibt unterschiedliche Nutzungsweisen von Kernwaffen: Sie können der Machtdemonstration dienen oder mit ihrem Einsatz wird gedroht, um US-Verbündete einzuschüchtern und unter Druck zu setzen. Letzteres dient dazu, Druck auf die USA auszuüben, um zu verhindern, dass Washington eskaliert.

Dies bedeutet, dass für Russland und China ihre Atomstreitkräfte und ihre strategischen Streitkräfte eine zentrale Rolle bei den regionalen Konflikten spielen, die uns hier interessieren: Mit ihnen soll verhindert werden, dass die Vereinigten Staaten oder deren Verbündete als Reaktion auf Angriffe Russlands oder Chinas in regionalen Konfliktsituationen den Weg der Eskalation gehen.

Daraus ergeben sich wichtige Folgen für die blaue Theorie des Sieges. Diese muss das Eskalationspotenzial und die Eskalationsstrategien von Russland und China berücksichtigen. Sie muss auch die spezifischen Aufgaben von Kernwaffen in deren Strategien berücksichtigen, insbesondere, wenn diese darauf abzielen, ihren Gegnern den Zugang zu gewissen Gebieten zu verwehren, sie zu einem bestimmten Verhalten zu zwingen, sie abzuschre- cken und zu besiegen. Aus US-amerikanischer Sicht gibt es einen wichtigen weiteren Parameter: die Tatsache, dass mehrere Operationsgebiete (z. B. Nordosteuropa oder Taiwan) zur selben Zeit betroffen sein können. Ein regionaler Krieg zwischen den Vereinigten Staaten und einer rivalisierenden Großmacht würde es erforderlich machen, militärische Ressourcen aus der ganzen Welt heranzuziehen. Der resultierende Abzug von Kräften aus anderen Einsatzgebieten, in denen diese auch Sicherheitsgarantien bieten, könnte von der anderen rivalisierenden Macht als eine Gelegenheit angesehen werden, dort Schaden zu stiften - entweder durch verstärkte Grauzonen-Aktivitäten oder eine direkte militärische Herausforderung der regionalen Ordnung. Russland und China müssen keine Verbündeten sein, um in dieser Weise zum Nachteil der USA zusammenzuwirken.

Hieraus folgt eine weitere wichtige Konsequenz für die blaue Theorie des Sieges. Diese muss hinreichende Abschreckungsmittel in dem vernachlässigten Operationsgebiet vorsehen. Joseph F. Dunford, der ehemalige US-Generalstabschef, brachte den Kern dieser neuen Herausforderung auf den Punkt, als er 2017 erklärte: „Mit hoher Wahrscheinlichkeit werden alle unsere Konflikte in Zukunft transregional, domänenübergreifend und multifunktional sein.“15 Der transregionale Aspekt kann auch bedeuten, dass ein Großmachtrivale den Krieg wahrscheinlich nicht auf ein einziges Konfliktgebiet beschränken würde und durchaus Angriffe auf überseeische Basen, die die US-Machtprojektion stützen, und auf kritische US-Infrastruktur und Führungseinrichtungen versuchen könnte. Der domänenübergreifende Aspekt ist eine Folge des Wettstreits um militärische Vorteile, der gegenwärtig zum Beispiel im Cyberspace, im Weltraum und in maritimen (und unterseeischen) Räumen ausgetragen wird. Der multifunktionale Aspekt wiederum rührt von dem breiten Spektrum militärischer Fähigkeiten und Aufgaben her, das bei einer Konfrontation mit einer Großmacht in Anspruch genommen würde. Konflikte von der Art, wie sie Dunford erwartet, haben ein erhebliches Eskalationspotenzial. Sie unterstreichen auch, wie wichtig die Fähigkeit der Vereinigten Staaten und ihrer Verbündeten ist, sämtliche Instrumente der Abschreckung und Kriegsführung bei Planungen und bei Einsätzen miteinzubeziehen und die Effekte in einer Weise aufeinander abzustimmen.

\footnotetext{
15 Thornhill/Karlin 2018.
} 


\subsection{Die Rolle des nuklearen „Schattens“}

Paul Nitze verglich in seinem 1956 erschienenen Buch über nukleare Abschreckung Kernwaffen mit der Dame auf einem Schachbrett, die einen langen Schatten auf das Brett und die Spielzüge wirft: „Unabhängig davon, ob Atomwaffen jemals in einem Krieg eingesetzt werden, beeinflusst die bloße Tatsache ihrer Existenz - die Möglichkeit, dass sie eingesetzt werden könnten - alle zukünftigen Kriege ... Die Situation entspricht derjenigen beim Schachspiel. Die atomaren Damen werden vielleicht nie ins Spiel gebracht; sie schlagen vielleicht niemals eine der Spielfiguren des Gegenspielers. Trotzdem hat die Position der atomaren Damen einen entscheidenden Einfluss darauf, welche Seite ohne Risiko einen Läufer des begrenzten Krieges oder einen Bauern des kalten Krieges vorwärtsbewegen kann. “16

Ungeachtet der Bestrebungen, Kernwaffen abzuschaffen, hat es sich bislang als unmöglich erwiesen, die Bedingungen zu schaffen, unter denen sich atomar bewaffnete Staaten sicher genug fühlten, um auf diese Waffen zu verzichten. ${ }^{17}$ Der Schatten liegt also weiterhin auf jedem Konflikt mit einem atomar bewaffneten Gegner. Obgleich das Risiko von Armageddon-Kriegen unterschwellig immer vage mitschwingt, ist es vordergründig von dem Risiko regionaler Kriege unter dem nuklearen Schatten abgelöst worden. ${ }^{18}$ Präsident Putin sieht in Kernwaffen ein Instrument, um Russland zu alter Bedeutung und zum Großmachtstatus zurückzuführen; ja, er räumt ihnen einen geradezu sakralen Status ein und betrachtet sie als Eckpfeiler seiner Strategie des „harten Zurückschlagens.“" Das russische Militär hat sich eine Doktrin und eine Streitkräftestruktur gegeben, die es ihm erlaubt, bei regionalen Konflikten Kernwaffen mit dem Ziel einzusetzen, durch kontrollierte Eskalation die Oberhand zu gewinnen. Auch China dürfte, nachdem es Kernwaffen ausdrücklich zu dem Zweck beschafft hat, „nukleare Drangsalierung zu durchkreuzen“, wohl kaum bereit sein, zu einer Zeit wachsender Sorge über Schikanen durch die USA solche Waffen aufzugeben. Die Weigerung Nordkoreas, auf seine Kernwaffen zu verzichten, sei hier ergänzend erwähnt.

Entsprechend müssen die Vereinigten Staaten, wie oben dargelegt, davon ausgehen, dass Kernwaffen in regionalen Kriegen gegen Russland oder China eine Rolle spielen. Das heißt nicht, dass sie notwendigerweise auch eingesetzt werden würden. Russland oder China werden

16 Nitze 1956

17 Roberts 2019.

18 Krause 2020.

19 Adamsky 2019. sie wahrscheinlich als Instrumente der Zwangsausübung, Erpressung und der Drohung mit äußerster Risikobereitschaft (brinkmanship) nutzen. Ihr tatsächlicher Einsatz in kriegerischen Konflikten erscheint unwahrscheinlich, auch wenn ein begrenzter Einsatz als ein akzeptables Risiko erachtet werden könnte. Die Androhung derartiger Angriffe soll aber politische Entschlossenheit signalisieren und die Vereinigten Staaten und/oder ihre Alliierten dazu bringen, klein beizugeben.

Unter diesen Umständen ist davon auszugehen, dass auch die Vereinigten Staaten ihre Atomstreitkräfte mobilisierten, um ihren Verbündeten zu versichern, dass sie keine schwerwiegenden Bedrohungen ihrer vitalen Interessen zuließen. Dadurch signalisierten auch sie ihre Entschlossenheit. Tatsächlich einsetzen würden sie diese aber nur unter extremen Umständen - nämlich dann, wenn ihre vitalen Interessen oder die eines Verbündeten akut bedroht wären. Allerdings werfen nicht nur Kernwaffen den Schatten der Eskalation. Neue Technologien haben neue Mittel geschaffen, um über große Entfernungen und mit potenziell unvermittelten Wirkungen möglicherweise einen entscheidenden Einfluss auf einen Feind auszuüben. Sowohl der Cyberspace als auch der Weltraum sind mittlerweile militärische Domänen. In beiden geht der Wettstreit weiter, da jeder der Rivalen eine Form von Dominanz oder Überlegenheit anstrebt. Die potenziell in, von oder durch diese Domänen erzeugten Effekte könnten die politische Entschlossenheit und die Effektivität von Einsätzen stark beeinflussen. Andererseits könnten diese Effekte in einem kleineren Maßstab auch nützlich sein, da sie signalisieren, wer besser gewappnet ist, um die Initiative zu ergreifen, wenn plötzlich ein Krieg ausbräche. Die Anwendung Künstlicher Intelligenz für diese Zwecke dürfte sowohl den wahrgenommenen Nutzen des Wettstreits und von Operationen in diesen Domänen steigern als auch herkömmliche Sichtweisen von strategischer Stabilität und ihre praktische Umsetzung obsolet machen. Die potenziellen Wechselwirkungen dieser neuen Technologien mit dem nuklearen Faktor sind äußerst beunruhigend.

Diese neuen Domänen steigern die Komplexität der neuen strategischen Herausforderung für die Vereinigten Staaten, weil Veränderungen in diesen Domänen die Entscheidungen feindlicher Politiker und Militärführer über die Initiierung oder Fortführung eines Konfliktes maßgeblich beeinflussen können. Operationen im Cyberspace und im Weltraum haben den potenziell einzigartigen Vorteil, dass sie eine Zwangswirkung in einer Weise entfalten, die nur für die politisch Verantwortlichen auf der anderen Seite erkennbar sind, nicht für die allgemeine Bevölkerung. Dies ermöglicht es, dass man sich „ungesehen“ 
nötigen lässt, was den öffentlichen politischen Preis einer nachgiebigen Haltung verringert.

Wenn man die Komplexität der neuen strategischen Herausforderung für die Vereinigten Staaten betrachtet, stechen zwei weitere Faktoren besonders hervor: Russland und China betrachten ihre Konflikte mit den Vereinigten Staaten im Wesentlichen als Nullsummenspiele. Als revisionistische Mächte sind sie bemüht, die von den USA gestützten regionalen Sicherheitsordnungen in ihren jeweiligen Nachbarschaften zu beseitigen und eine neue Ordnung zu schaffen, die sich mit ihren Präferenzen deckt. Wenn ihnen dies gelänge, hätte dies weitreichende Folgen über das lokale Machtgleichgewicht hinaus. Es würde für ihre Nachbarn erhebliche Souveränitätseinbußen bedeuten. Sie müssten ständig Rücksichtnahme gegenüber den mutmaßlichen Interessen Russlands und Chinas üben. Demokratische Institutionen, Praktiken und Normen verblassten oder verschwänden. Es käme zu einer Neugestaltung der weltwirtschaftlichen Ordnung, die für die Vereinigten Staaten und ihre Verbündeten nachteilig wäre. Die Folge wäre eine USA, die isoliert, verwundbar und noch tiefer gespalten wäre. Anders gesagt: Bei diesem Konflikt steht für die Vereinigten Staaten und ihre Verbündeten sehr viel auf dem Spiel, und die Reaktion darauf sollte entsprechend sein.

Ein Krieg zwischen den Vereinigten Staaten und Russland oder China hätte zudem weitreichende Folgen für viele weitere Akteure im internationalen System. Ein solcher Krieg würde um die Natur der regionalen Ordnungen in Europa und in Asien geführt werden, und es könnten auch Kernwaffen in ihm eingesetzt werden. Er wäre der entscheidende Test für den Willen und die Fähigkeit der Vereinigten Staaten, ihre Verpflichtungen als ein Sicherheitsgarant für andere zu erfüllen und eine internationale Ordnung aufrechtzuerhalten, die sie nach dem Zweiten Weltkrieg selbst mitaufgebaut und während des Kalten Krieges und darüber hinaus mitgetragen haben.

\section{Warum eine Theorie des Sieges sinnvoll ist}

$\mathrm{Zu}$ einem Zeitpunkt, an dem die Vereinigten Staaten sich in Afghanistan und im Irak auf andere strategische Herausforderungen konzentrierten, trafen Russland und China umfangreiche Vorbereitungen für einen möglichen regionalen Krieg mit den Vereinigten Staaten und ihren Verbündeten. Es ist der Versuch, einen breit angelegten, mehrdimensionalen Konflikt mit den Vereinigten Staaten vorzubereiten, der militärische, politische, wirtschaft- liche und ideologische Aspekte einschließt und darauf abzielt, die regionalen Ordnungen und auch die Weltordnung umzugestalten. Wir müssen daher die Theorien des Sieges unserer Gegner verstehen und eigene Theorien entwickeln. Dabei sollten wir uns auf eine Reihe plausibler Grundsätze stützen, die es uns erlauben, einen Feind zu bezwingen. Dagegen könnte man einwenden, dass eine Theorie des Sieges unnötig sei, weil der „Sieg“ bereits von dem Begriff „Strategie“ umfasst werde. Dieser Gedanke ist - wie oben schon ausgeführt worden ist - falsch. Die Einwände ermahnen uns aber, die Anforderungen an eine robuste Theorie genau zu definieren.

Erinnern wir uns auch an die rhetorische Kraft des Siegeskonzepts. Diese Kraft besitzt einen eigenen Wert. Regierungen, die eine Nation in den Krieg führen, haben eine moralische Verpflichtung, denjenigen, von denen sie Opfer verlangen, zu erklären, warum die Sache es wert sei, Leib, Leben und Besitz zu opfern. Dies gilt in besonderem Maße für Kriege mit erheblichem Eskalationspotenzial. Diejenigen, von denen Opfer verlangt werden, müssen verstehen, dass etwas Bedeutendes auf dem Spiel steht und dass die Opfer, die sie bringen, entscheidend dazu beitragen, einen grundlegenden Daseinszweck der Nation zu erfüllen. Sie müssen verstehen, dass die militärische Kampagne, deren Unterstützung von ihnen verlangt wird, sowohl notwendig als auch verdienstvoll ist. Daraus folgt die Notwendigkeit, ein Konzept des Sieges zu definieren, das weit über den Aspekt der „Bestrafung“ hinausgeht.

Damit verbunden ist eine moralische Verpflichtung, eine Vision des Sieges vorzulegen, die einen dauerhaften Frieden verspricht. Eine grundlegende Voraussetzung für einen solchen Frieden besteht darin, dass er gerecht sein muss und Streitfragen friedlich und nachhaltig beilegt. Anders ausgedrückt: Diejenigen, von denen Opfer verlangt werden, erwarten zu Recht, dass in der Vision des Sieges die Voraussetzungen für einen gerechten Krieg und einen gerechten Frieden dargelegt werden. Wenn man den Sieg - statt den Erfolg - in den Vordergrund rückt, muss man das Vermächtnis der in den 1980er-Jahren geführten Debatte über die Frage, ob es möglich sei, einen umfassenden Atomkrieg zu gewinnen beziehungsweise ob einer solcher Sieg nicht sinnlos wäre, hinter sich lassen. Stattdessen sollte man sich auf die Frage konzentrieren, wie in einem Konflikt mit einem Gegner, der willens und fähig ist, nukleare Bedrohungen anzuwenden, um US-Verbündete und die Vereinigten Staaten unter Druck zu setzen und der dabei bereit ist, bis zum Äußersten zu gehen und vielleicht sogar begrenzte Atomschläge auszuführen, die eigenen Interessen gewahrt werden können. Dies bedeutet, dass die blaue Theorie des Sieges ein überzeugendes Konzept für den angemessenen und besonnenen Einsatz 
von Kernwaffen beinhalten muss, um einen Konflikt möglichst rasch $\mathrm{zu}$ beenden. Ohne eine solche Theorie des Sieges dürften die Risiken steigen, dass die rote Seite „nuklear abgesicherte“ Aggressionen initiiert. Man muss dies gesondert von der Frage betrachten, ob ein totales nukleares Armageddon zu gewinnen sei - was unbestreitbar nicht der Fall ist. Die Diskussion über das Konzept der Theorie des Sieges trägt somit auch dazu bei, die Grenzen dieser Überlegungen zu verdeutlichen. Eine Theorie des Sieges umfasst nicht alle Elemente der Entwicklung strategischer, taktischer und operativer Pläne. Frank Hoffman weist zu Recht darauf hin, dass eine Theorie des Sieges „kein Patentrezept für strategische Kompetenz ist, die viele Elemente umfasst, aber sie ist von zentraler Bedeutung für strategischen Erfolg. “20

\subsection{Kernelemente einer blauen Theorie des Sieges}

Wenn man eine blaue Theorie des Sieges entwickeln will, muss man sich an die Kernelemente der roten Theorie des Sieges erinnern:

1. Wenn ein Krieg mit den Vereinigten Staaten unvermeidlich erscheint, ist es notwendig und möglich, möglichst rasch vollendete Tatsachen $\mathrm{zu}$ schaffen und einen massiven US-Gegenschlag zu verhindern, indem man die USA und ihre Verbündeten auf ihre Schwachstellen verweist.

2. Wenn die Vereinigten Staaten den Versuch unternehmen sollten, den Status quo ante durch den Einsatz regulärer Streitkräfte wiederherzustellen, kann dies erfolgreich dadurch unterbunden werden, dass man ihre Verbündeten durch Drohungen und Angriffe von den Vereinigten Staaten abspaltet oder auch untereinander trennt.

3. Sofern diese Anstrengungen scheitern, können militärische Operationen der USA durch kinetische und nicht kinetische Angriffe so kostspielig gemacht werden, dass sie ihre Kriegsziele nicht weiterverfolgen. Im Extremfall könnten kinetische Angriffe auch Angriffe mit nicht strategischen Kernwaffen umfassen.

4. Wenn diese Bemühungen nicht zu einer zeitnahen Beendigung des Krieges führen und sich eine bedeutende neue Bedrohung auftut, wird es die Vereinigten Staaten dazu bringen, sich mit der Niederlage abzu-

20 Hoffman 2020, 56. finden, wenn man sie daran erinnert, wie verwundbar ihr eigenes Territorium für Angriffe ist.

Diese Theorie basiert auf Zwangsanwendung, Erpressung und Androhung und auf der Bereitschaft, bis zum Äußersten zu gehen. Es geht um die Erreichung spezifischer Ziele, wobei ein Krieg begrenzt gehalten werden soll. Sie stellt die Vereinigten Staaten und ihre Verbündeten bei wichtigen Entscheidungspunkten vor die Wahl zwischen Deeskalation und riskanter Eskalation. Jeder dieser Punkte ist ein „Kulminationspunkt“ im Sinne von Clausewitz - der Punkt, an dem eine Entscheidung zwischen den Kosten und den Risiken einer Fortsetzung des Krieges und dem, was dabei auf dem Spiel steht, getroffen werden muss.

Welches Grundprinzip sollte die blaue Theorie des Sieges leiten? Eine Reihe von Konzepten steht zur Diskussion. Eines ist das klassische Konzept der Abschreckung: Zweifellos wollen die Vereinigten Staaten Russland und China von Aggressionen und, im Falle eines Krieges, von deren Eskalation abhalten. Aber als ein strategisches Ordnungskonzept ist der Nutzen dieses Konzepts durch seine enge Verbindung mit der Nuklearstrategie und durch die Assoziation mit dem Kalten Krieg begrenzt. Außerdem bringt das Abschreckungskonzept kaum neue Denkanstöße bezüglich der Frage, wie man einen Krieg gewinnen kann.

Ein alternatives Grundprinzip ist Eskalationskontrolle. Es wäre schön, wenn man eine solche Kontrolle hätte, aber gegenüber einer Großmacht mit Kernwaffen gibt es sie schlichtweg nicht. Selbst in dem seltenen Fall, in dem die Vereinigten Staaten vielleicht die technischen Mittel besäßen, um dies in einem engen, militärisch-operativen Sinne zu erreichen, könnten sie doch kaum feindliche Gegenschläge verhindern, die mit hohen Kosten für die USA verbunden wären. ${ }^{21}$

Ein besseres Leitprinzip ist es, wenn man die Vorgehensweise von Rot versteht und Rot zu Entscheidungen darüber zwingt, wie hohe Risiken und welche Kosten es zu tragen bereit wäre, sollte Rot versuchen, die Vereinigten Staaten und ihre Verbündeten zu jenem oben beschriebenen Entscheidungspunkt zu bringen. So wie Rot danach strebt, Blaus Kalkül von Nutzen, Kosten und Risiken zu beeinflussen, so sollte Blau das Kalkül von Rot zu beeinflussen versuchen - und zwar effektiver. Man könnte von einem „Eskalationskalkül“ sprechen. Die Vereinigten Stabschefs der USA beschreiben normalerweise ein „Abschreckungskalkül,“ das aus dem Kalkül des Feindes über den Nutzen einer bestimmten Handlungsweise,

21 Fitzsimmons 2017. 
deren Kosten und Risiken und den potenziellen Vorteilen, Kosten und Risiken möglicher anderer Handlungsweisen einschließlich Untätigkeit besteht. ${ }^{22}$ Dieses nützliche Konstrukt passt auch gut zu dem roten und blauen Eskalationskalkül. Jede Seite will die von der anderen Seite vorgenommene Einschätzung der Vorteile, Kosten und Risiken verschiedener Handlungsweisen an zentralen Entscheidungspunkten beeinflussen.

Das Kernkonzept der blauen Theorie des Sieges sollte daher, kurz gesagt, darin bestehen, Zwangsanwendung dadurch zu kontern, dass man das Vertrauen von Rot in sein Eskalationskalkül untergräbt. Eine solche Vorgehensweise hätte den großen Vorteil, dass sie das Vertrauen von Blau in sein Eskalationskalkül stärkte. Wenn diese Vorgehensweise erfolgreich wäre, erlaubte sie es den Vereinigten Staaten, die Initiative zu ergreifen und diese zu behalten, die Kosten und Risiken einer Eskalation auf Rot zu verschieben, und den Feind zu zwingen, die Bedingungen der USA für eine Einstellung der Feinseligkeiten zu akzeptieren.

Dieser Ansatz lässt sich mit den folgenden Hypothesen auf das oben erwähnte allgemeine Problem anwenden:

1. Blau kann den Versuch, ein fait accompli zu schaffen, dadurch vereiteln, dass es ein konventionelles Abschreckungsmittel schafft, das nicht leicht überwunden werden kann und das, falls es angegriffen würde, bei der anschließenden Reaktion viele Akteure einbezöge. Blau sollte glaubhaft versichern, dass ein Angriff auf die vitalen Interessen eines Verbündeten für Rot die gleichen Risiken mit sich brächte wie ein Angriff auf vitale Interessen der USA. Außerdem kann es die Effekte angedrohter Raketenangriffe durch ein Raketenabwehrsystem vermindern. Kurzum, ein verbessertes konventionelles Abschreckungsmittel mit verbesserter Angriffsfähigkeit würde die erwarteten Kosten von Rot erhöhen, während Raketenabwehrsysteme den erwarteten Nutzen einer Zwangsmaßnahme für Rot verringern würde.

2. Verbündete müssen ausreichend rückversichert werden, sodass sie sich an amerikanischer Seite an Truppenbewegungen und Einsätzen beteiligen. Sie können auch ihre eigene Entschlossenheit demonstrieren, sich nicht durch Zwangsmittel von der Verfolgung ihrer Interessen abbringen $\mathrm{zu}$ lassen. Rot könnte darauf mit intensivierten Angriffen antworten, müsste aber mit dem Risiko rechnen, dass die US-Ver-

22 U.S. Joint Chiefs of Staff: Strategic Deterrence Joint Operating Concept, Washington, D.C., December 2006. bündeten ihrerseits Druck auf die Vereinigten Staaten ausübten mit dem Ziel, einen kriegsentscheidenden Gegenschlag zu führen, was wiederum das Risiko für Rot erhöhen würde.

3. Blau kann seine Fähigkeit zur Machtprojektion aufrechterhalten, indem es Ressourcen in seinen Verladeund Entladehäfen besser schützt, mehr räumlich weit auseinanderliegende Operationen durchführt und inländische kritische Infrastruktur besser verteidigt. Blau kann zudem regionale Raketenangriffe massiv vergelten, in einer Weise, die Rot von einer weiteren Eskalation abhalten sollte.

4. Rot kann durch eine glaubhafte Drohung mit einem Gegenschlag vom Einsatz von Kernwaffen abgeschreckt werden. Sollte diese Abschreckung scheitern und Rot einen begrenzten Atomwaffenangriff durchführen, könnte eine angemessene Reaktion darauf Rot verdeutlichen, dass es die Entschlossenheit von Blau falsch eingeschätzt hat. Dies würde nicht zu einer weiteren nuklearen Eskalation durch Rot führen, weil Rot einsähe, dass solche Angriffe neue Anreize für Blau schaffte.

5. Die Vereinigten Staaten können Angriffe auf ihr Territorium mit einer Mischung aus defensiven und offensiven Fähigkeiten und klaren Drohungen abschrecken.

6. Durch verbesserte Resilienz im Cyberspace und im Weltraum können die Vereinigten Staaten und ihre Verbündeten den erwarteten Nutzen von Angriffen für Rot verringern. Verbesserte Angriffsfähigkeiten der USA und ihrer Alliierten in diesen Domänen würden die erwarteten Kosten von Rot in die Höhe treiben.

7. Die vermeintliche Asymmetrie dessen, was für jede Seite auf dem Spiel steht, die Rot begünstigen soll, darf nicht aufkommen. Ein „erfolgreicher“ regionaler konventioneller Krieg eines atomar bewaffneten Gegners gegen einen Verbündeten der USA würfe grundlegende Fragen bezüglich der Glaubwürdigkeit der USA als Sicherheitsgarant auf. Dabei steht sehr viel auf dem Spiel. Die Vereinigten Staaten müssen wirksam kommunizieren, worum es ihnen geht.

8. Die Asymmetrie der Regierungsführung spielt Blau, nicht Rot, in die Hände. Ungeachtet ihrer Reputation als „starke Männer“ fehlt es den Staatsführern in Russland und China an politischer Legitimität, da ihre Macht zum Teil auf den Gewehrläufen und auf anderen Mitteln der Zwangsanwendung basiert. Ihre (mangelnde) Legitimität wird bei einer nationalen Krise offenkundig, die mit hohen Kosten und Risiken verbunden ist. Der demokratische Charakter von Blau ist eine Quelle strategischer Stärke, weil ein Angriff eine gespaltene Bevölkerung in der Regel zusammen- 
schweißt und weil ihre Einheit für die Entschlossenheit sorgt, die notwendig ist, um einen Aggressor zu besiegen.

9. Die Asymmetrie der geografischen Lage muss nicht von Belang sein. Jeder größere Angriff auf US-Streitkräfte oder auf einen US-Verbündeten zöge immer eine kraftvolle US-Reaktion nach sich. Eine weitere Eskalation von Rot durch Angriffe auf US-Territorium würde für die Vereinigten Staaten die Natur des Krieges verändern, da für sie nun erheblich mehr auf dem Spiel stünde. Dies würde eine massive Reaktion auf den Angreifer rechtfertigen.

Ein Sieg für Blau in einer Krise oder in einem Krieg sollte daher definiert werden als Bewahrung der Souveränität und Sicherheit der Vereinigten Staaten und ihrer Verbündeten, als Bewahrung der regionalen Sicherheitsordnung und Erhaltung der Integrität der Rolle der USA als Sicherheitsgarant. Der Sieg erfordert es, dass man einen bewaffneten Herausforderer an einen Kulminationspunkt bringt, eher früher als später, an dem er zu der Einschätzung gelangt, dass die Kosten und Risiken einer Fortsetzung des von ihm begonnenen Krieges untragbar sind. Dieser Theorie zufolge müssen die politisch Verantwortlichen bei Rot an Entscheidungspunkte gebracht werden, denen sie lieber ausweichen würden, während Blau sich seinerseits den gleichgerichteten Bemühungen von Rot entziehen müsste. Nur so kann das Vertrauen von Rot in sein Eskalationskalkül untergraben werden.

In dieser Theorie des Sieges würden die Vereinigten Staaten die Eskalation nicht kontrollieren. Sie würden vielmehr ihrem Gegner stets Kosten und Risiken auferlegen, um sein Eskalationskalkül zu beeinflussen oder gar zu durchkreuzen. Reaktionen des Gegners würden nicht kontrolliert. Sie kämen durch Zwang oder durch Anreize zustande. Nicht alle Formen der Eskalation von Blau würden dieses Kalkül möglicherweise entscheidend beeinflussen. Die konkreten Eskalationsschwellen müssen sich an Entscheidungspunkten befinden, an denen ein Gegner gezwungen ist, zwischen zwei Handlungsweisen zu wählen: einer, die nicht das gewünschte Ergebnis bringt und die kostspieliger und riskanter wird, und einer, die noch gewisse Interessen schützt, auch wenn sie den Sieg verfehlt. Dies ist eine Antieskalationsstrategie im Gegensatz zu einer Eskalationskontrollstrategie.

Eine zentrale, unbeantwortete Frage in dieser Rahmentheorie von Blau lautet: Muss Rot bestraft werden, nachdem es besiegt worden ist? Diese Frage wäre besonders heikel in einer Situation, in der Rot Kernwaffen eingesetzt hat. Würden die Opfer einer nuklear gestützten Aggression oder eines nuklearen Angriffs eine Rückkehr zum Status quo ante nach dem Krieg als ein akzeptables Ergebnis betrachten? Würde der Status quo ante als eine brauchbare Grundlage für eine dauerhafte und gerechte Beilegung der Streitfrage angesehen? Wenn die Antwort auf beide Fragen „Nein“ lautet, dann müsste Blau nach einem Weg suchen, Rot zu bestrafen, ohne Rot anzuspornen, seinen Einsatz von Kernwaffen fortzusetzen oder gar noch auszuweiten. Im konkreten Fall mag Blau die Mittel dazu haben oder auch nicht. Der Theorie zufolge wird Blau das damit verbundene Dilemma nicht lösen können, wie eine Bestrafung erfolgen soll, wenn die andere Seite auch über Kernwaffen verfügt. Es ist davon auszugehen, dass Blau dann keine Bestrafung vornähme.

\section{Die Anwendung des Modells auf spezifische Regionen}

Diese allgemeinen Konzepte müssen in ihre regionalen Kontexte übersetzt werden. Beginnen wir mit Europa, ehe wir uns Nordostasien zuwenden.

\subsection{Ein theoretischer Rahmen für Europa}

Während Russland eine umfassende Strategie für regionale, domänenübergreifende Zwangsanwendung und für einen Krieg gegen die NATO besitzt, hat die NATO eine allgemeine Abschreckungsstrategie und eine umfassende Abschreckungs- und Verteidigungsdoktrin. Die allgemeine Abschreckungsstrategie ergibt sich aus dem grundlegenden Selbstverständnis der NATO als einer „Allianz ohne Feinde, “ auch wenn sie ein paar schwierige Nachbarn hat. ${ }^{23}$ Die umfassende Abschreckungs- und Verteidigungsdoktrin umfasst eine „geeignete Mischung“ nuklearer und nicht nuklearer Fähigkeiten zur Abschreckung, die, so verspricht die Allianz, angepasst werde, um „zwecktauglich“ zu bleiben, während sich die sicherheitspolitischen Rahmenbedingungen wandelten. ${ }^{24}$ Im Anschluss an die bewaffnete Annexion der Krim beschloss die NATO auf ihrem Gipfel in Wales im Jahr 2014 Maßnahmen, um die konventionelle Abschreckung entlang ihrer nördlichen Flanke und den Schutz gegen russische Techniken

23 NATO: Active Engagement, Modern Defence: Strategic Concept for the Defence and Security of the Members of the North Atlantic Treaty Organization 2010; https://www.nato.int/cps/en/natolive/official_ texts 68580.htm.

24 NATO: Deterrence and Defense Posture Review 2012; https://www. nato.int/cps/en/natolive/official_texts_87597.htm. 
der hybriden Kriegsführung zu stärken. Zwei Jahre später traf die NATO in Warschau weitere Beschlüsse, um ihre Abschreckung an neue domänenübergreifende Herausforderungen anzupassen, die von Russland ausgehen, und um ihre nukleare Abschreckungsstrategie zu stärken. Seit dem ersten Gipfel der Trump-Ära konzentriert sich die NATO darauf, ihre Abschreckungsdoktrin weiterzuentwickeln und zu stärken.

In die Debatte zwischen NATO-Experten darüber, wie man am besten auf die russischen Kriegsvorbereitungen reagieren sollte, gingen in den letzten Jahren ein paar Ideen ein, auf deren Grundlage eine Siegestheorie der NATO in Krisen- und Kriegszeiten konzipiert werden könnte. ${ }^{25}$ Diese enthielte die folgenden Prämissen:

1. Wenn eines oder mehrere ihrer Mitglieder angegriffen werden, wird die Allianz geeint und entschlossen sowohl den Verbündeten als auch den Grundsatz verteidigen, dass ein Angriff auf einen ein Angriff auf alle ist. Versuche, Zwang anzuwenden, sowie begrenzte Machtdemonstrationen werden diese Entschlossenheit verstärken.

2. Ein verlockender konventioneller fait accompli kann dadurch weniger verlockend gemacht werden, dass die konventionelle Abschreckung an der Nordflanke der NATO gestärkt wird, wo eine multinationale Streitmacht (zu der Truppen aus allen drei atomar bewaffneten NATO-Mitgliedern gehören) als Stolperdraht fungiert, der Russland zwänge, Soldaten aus vielen Ländern zu töten, mit der Aussicht auf einen massiven Gegenschlag. Diese Maßnahmen wurden weitgehend schon beschlossen.

3. Jeder Versuch, die Vereinigten Staaten durch Drohungen oder begrenzte Angriffe auf US-Territorium von der Verteidigung Europas abzukoppeln, muss zum Scheitern verurteilt werden, weil die Vereinigten Staaten ein bleibendes Interesse an einem stabilen, friedlichen Europa haben. Außerdem führten solche Angriffe nur dazu, dass sich in der amerikanischen Öffentlichkeit und Politik ein aus gemeinsamen historischen Erfahrungen und geteilten Werten gespeistes Gefühl der Verbundenheit mit Europa regte.

4. Moskau hat zahlreiche wirtschaftliche, politische und militärische Gründe dafür, einen aus seiner Sicht schlecht verlaufenden Konflikt in Europa nicht zu eskalieren. Aber wenn es dies trotzdem - in vertikaler oder horizontaler Richtung - tun sollte, muss die NATO mit ihren vielfältigen kinetischen und nicht kinetischen Angriffsfähigkeiten die Führungsriege in

25 Kulesa 2018.
Moskau derart aufrütteln, dass sie ihre gravierende Fehleinschätzung erkennt. Die NATO kann auch den von Russland erwarteten Nutzen begrenzter kinetischer und nicht kinetischer Angriffe auf den Westen durch einen begrenzten Raketenschutzschild und Resilienz im Cyberspace und im Weltraum verringern.

5. Russland würde die Schwelle zu einem großen Atomkrieg in Europa oder zu einem uneingeschränkten strategischen Krieg gegen die Vereinigten Staaten nicht überschreiten, weil die drei nuklear bewaffneten NATO-Mitglieder glaubhafte strategische Abschreckungsmittel besitzen.

6. Russland würde die Schwelle zu einem begrenzten Atomkrieg in Europa nicht überschreiten, weil es (a) eine Eskalationsspirale befürchtet und (b) die Vereinbarungen zur nuklearen Teilhabe der NATO auf wirkungsvolle Weise die kollektive nukleare Entschlossenheit der Allianz signalisieren und eine begrenzte, taktische Vergeltung für jeden begrenzten russischen Angriff mit einer taktischen Kernwaffe sicherstellen.

7. Falls Russland die nukleare Schwelle überschreiten sollte, würde es dies in einem ersten Schritt vermutlich nur in einer begrenzten Weise tun. Seine weitere Eskalation könnte durch eine angemessene Reaktion der NATO verhindert werden, die unter Beweis stellt, dass sie auch weiterhin in der Lage ist, ihre Interessen zu verteidigen. Durch die Vorbereitung der Nachführung zusätzlicher Kräfte sollte sie ihre entsprechenden Fähigkeiten sichtbar werden lassen.

8. Allen NATO-Mitgliedern muss bewusst sein, dass für alle bei einem Krieg mit Russland nicht weniger auf dem Spiel steht als für Russland. Tatsächlich ginge es für die NATO sogar um noch mehr als für Russland, denn bei einem begrenzten Verteidigungskrieg würden erst einmal nur die Souveränität und die Integrität einiger weniger NATO-Mitgliedstaaten in Frage gestellt. Die Souveränität und Integrität Russlands steht erst einmal nicht auf dem Spiel. Außerdem ist es wichtig, dass die Glaubwürdigkeit der globalen Sicherheitsgarantie der USA bewahrt bleibt.

Um es zu wiederholen: Dies ist eine Sammlung von Ideen, die innerhalb der Allianz gelegentlich diskutiert werden. Sie sind nicht an einem Ort niedergeschrieben. Sie wurden von der Allianz jedenfalls nicht zu einer kohärenten Theorie des Sieges verknüpft. Wie würde eine Siegestheorie aussehen, wenn sie in eine NATO-Strategie für Konflikte mit Russland eingebettet würde? Übersicht 1 verdeutlicht dies unter Verwendung des gängigen Analysekonstrukts „Ziele-Methoden-Mittel.“ Sie zeigt, dass es notwendig ist, die potenziellen nächsten Eskalationsschritte Russlands 
Übersicht 1: Eine umfassende regionale Abschreckungsarchitektur

\begin{tabular}{|c|c|c|c|c|c|c|}
\hline $\begin{array}{l}\text { Rote } \\
\text { Schwellen }\end{array}$ & $\begin{array}{l}\text { Konventionelle } \\
\text { Kräfte }\end{array}$ & Raketenabwehr & Cyber & $\begin{array}{l}\text { Nicht-nukleare } \\
\text { Prompt Strikes }\end{array}$ & $\begin{array}{l}\text { Nukleare Teilhabe } \\
\text { der NATO }\end{array}$ & $\begin{array}{l}\text { Strategische } \\
\text { Nuklearkräfte } \\
\text { USA, F, UK }\end{array}$ \\
\hline $\begin{array}{l}\text { Versuchter fait } \\
\text { accompli }\end{array}$ & $\begin{array}{l}\text { Schnellen Erfolg } \\
\text { verweigern, trip } \\
\text { wire-Effekt }\end{array}$ & $\begin{array}{l}\text { Die Erfolgsaussicht } \\
\text { eines entschei- } \\
\text { denden Effekts } \\
\text { verringern }\end{array}$ & & & & \\
\hline $\begin{array}{l}\text { Erweiterte konven- } \\
\text { tionelle Attacke }\end{array}$ & & $\begin{array}{l}\text { Die Effekte von be- } \\
\text { grenzten Angriffen } \\
\text { begrenzen }\end{array}$ & \multicolumn{3}{|c|}{$\begin{array}{l}\text { Erwarteten Preis des Angriffs erhöhen und Risiko- } \\
\text { kalkulation erschweren }\end{array}$} & \\
\hline $\begin{array}{l}\text { Erweiterte Attacke } \\
\text { mit Cyber und in } \\
\text { Weltraum }\end{array}$ & & \multicolumn{4}{|c|}{$\begin{array}{l}\text { Vertrauen der anderen Seite in Leistung der A2/AD Systeme begrenzen und } \\
\text { Initiative übernehmen }\end{array}$} & \\
\hline $\begin{array}{l}\text { Nukleare (De-) Eska- } \\
\text { lation }\end{array}$ & & $\begin{array}{l}\text { Vertrauen in den } \\
\text { Erfolg verweigern, } \\
\text { dabei nüchtern } \\
\text { bleiben }\end{array}$ & & & $\begin{array}{l}\text { Kollektive Entschlos- } \\
\text { senheit unter Beweis } \\
\text { stellen }\end{array}$ & \\
\hline Nukleare Angiffe & & & & & $\begin{array}{l}\text { Drohung mit } \\
\text { begrenzter Vergel- } \\
\text { tung }\end{array}$ & $\begin{array}{l}\text { Signifikante } \\
\text { Risikoschwelle }\end{array}$ \\
\hline $\begin{array}{l}\text { Nuklearangriffe auf } \\
\text { US Territorium }\end{array}$ & & & & & & $\begin{array}{l}\text { Einmalig glaub- } \\
\text { würdige Drohung }\end{array}$ \\
\hline
\end{tabular}

im Voraus zu bedenken. Wenn man auf der Eskalationsleiter eine Sprosse nach der anderen hinaufsteigt, erlegt man dem Gegner (stufenweise steigende) Kosten und Risiken auf. Amerikanische Experten betrachten oftmals nur den Aspekt der Strategie, der mit der Auferlegung von Kosten verbunden ist.

Man sollte auch bedenken, dass bislang im Rahmen einer NATO-Endspielstrategie die Bestrafung Russlands kein Thema gewesen ist. NATO-interne Beratungen über die Notwendigkeit, Russland zu bestrafen, wären wahrscheinlich höchst strittig. Einige erachteten eine Bestrafung als notwendig, um den Frieden längerfristig $\mathrm{zu}$ sichern, während andere der Meinung wären, eine solche stachele nur russische Ressentiments an. Die Verantwortlichen der NATO müssten in dieser Situation eine Entscheidung treffen, die wahrscheinlich davon abhinge, für wie gravierend die Schäden eingeschätzt würden, die russische Streitkräfte verursacht beziehungsweise erlitten haben. Entscheidend wäre auch die Bereitschaft der Moskauer Führung, ihr Projekt einer grundlegenden Neugestaltung der europäischen Sicherheitsordnung aufzugeben, nachdem ihre Aggression gescheitert ist.

Da es letztlich darum geht, den Frieden zu gewinnen, wird daher eine ausgewogene Vorgehensweise gegenüber Russland erforderlich sein. Sie muss die politisch-mili- tärische Vorbereitungen zum Zweck der Abschreckung mit politisch-diplomatischen Maßnahmen zum Abbau von Spannungen und zur Verbesserung der politischen Beziehungen sowie mit Rüstungskontrolle und anderen Mitteln verbinden. Die im Harmel-Bericht der NATO von 1967 niedergelegten Grundsätze gelten auch heute noch: Die Allianz muss ausreichende militärische Stärke und politische Solidarität aufrechterhalten, um vor Aggressionen und anderen Formen von Druck abzuschrecken und um das Territorium von Mitgliedstaaten zu verteidigen, während sie sich gleichzeitig um eine tragfähigere Beziehung zu Russland bemühen sollte, in der grundlegende politische Streitfragen geklärt werden können. So hieß es im Harmel-Bericht: „Das oberste politische Ziel der Allianz besteht darin, eine gerechte und dauerhafte Friedensordnung in Europa zu verwirklichen, verbunden mit geeigneten Sicherheitsgarantien." ${ }^{26}$ Ob Regierende in Ost und West sich über das Erfordernis einer gerechten und dauerhaften Friedensordnung in Europa verständigen könnten, ist eine offene Frage (im Jahr 1945 erwies sich dies als unmöglich).

26 NATO: Report of the Council on the Future Tasks of the Alliance (1967); https://www.nato.int/cps/en/natohq/official_texts_26700. htm. 
Auch eine bloße Rückkehr zum Status quo ante würde nicht zufriedenstellen. Ein solcher Frieden würde erfordern, dass die eine oder die andere Seite ihr wichtigstes Anliegen aufgäbe. Es geht auf der einen Seite um das westliche Unterfangen, ein geeintes und freies Europa aufzubauen, auf der anderen Seite geht es um Präsident Putins Vorhaben, die bestehende westliche Ordnung zu zerstören und durch etwas zu ersetzen, was den russischen Interessen förderlicher ist. Solange es nicht zu einem Regimewechsel in Moskau - oder im Westen - kommt, erscheint eine politische Einigung unwahrscheinlich.

Um eine derartige Strategie $\mathrm{zu}$ beschließen und umzusetzen, benötigt die Allianz breitgefächerte Abschreckungs- und Verteidigungsfähigkeiten. Jahrzehntelang konnte die NATO bequem sagen (zum Beispiel in Gipfel-Communiqués), dass sie über den "geeignete[n] Mix“ konventioneller und nuklearer Abschreckungs- und Verteidigungsfähigkeiten verfüge. Aber in den letzten zehn Jahren hat sich das „Instrumentarium“ der Abschreckung und Verteidigung erweitert, denn es kamen Raketenabwehrsysteme und Fähigkeiten in den neuen militärischen Domänen Cyberspace und Weltraum hinzu. Übersicht 2 gibt dieses umfangreiche Instrumentarium und seine potenziellen Abschreckungswerte anschaulich wieder.

Ein zentrales, heikles und polarisierendes Thema innerhalb der NATO, wie auch andernorts, ist die nukleare Frage: Was ist die angemessene und notwendige Rolle von Kernwaffen in der Theorie des Sieges der NATO und welche Fähigkeiten sind notwendig, um diese Rolle zu erfüllen? Jahrzehntelange Diskussionen, Debatten und Kompromisse führten zu der - fragilen - Vereinbarung zwischen den Staats- und Regierungschefs der NATO, dass Kernwaffen in der Strategie der NATO eine Rolle spielten. Die NATO hat erklärt, dass sie so lange eine nukleare Allianz bleiben werde, wie es Kernwaffen gebe, und dass ihre Atomstreitkräfte ein wesentliches Element ihrer Abschreckungs- und Selbstverteidigungsstrategie und die ultimative Garantie der Souveränität und Integrität der Verbündeten seien. ${ }^{27}$

Aber hier geht es um die spezifische Frage, welchen Stellenwert Kernwaffen in der Siegtheorie der NATO haben sollen. Gegenüber einem atomar bewaffneten Gegner, der Kernwaffen eine zentrale Rolle zuschreibt, spielen die Atomstreitkräfte der NATO zwar begrenzte, aber einzigartige und bislang durch nichts anderes ersetzbare Rollen. Die grundlegende Rolle der Atomstreitkräfte der NATO in Krisenzeiten und im Falle eines Krieges mit Russ-

27 NATO: Strategic Concept 2010; https://www.nato.int/cps/en/ natohq/topics_82705.htm. land bestünde darin, das Vertrauen Moskaus darauf zu unterminieren, dass seine Kosten überschaubar blieben, wollte es die Souveränität eines NATO-Mitglieds bedrohen, die Integrität des Nordatlantikvertrags gefährden und zu diesen Zwecken Kernwaffen einsetzen oder damit drohen. Westliche Nuklearwaffen sollen daher Moskaus Vertrauen in die Berechenbarkeit der Risiken einer Aggression erschüttern.

Hier kommt die Frage der nuklearen Teilhabe ins Spiel. Die politisch Verantwortlichen in der NATO haben bislang ungeachtet erheblicher politischer Widerstände innerhalb der teilnehmenden Staaten an den einzigartigen Vereinbarungen zur nuklearen Teilhabe festgehalten. Sie taten dies hauptsächlich aus zwei Gründen. Die Teilhabe-Vereinbarungen erlauben es NATO-Mitgliedern zum einen, Moskau ihre kollektive Entschlossenheit zu demonstrieren, einer nuklearen Aggression zu widerstehen und den Grundsatz aufrechtzuerhalten, dass selbst beim angedrohten oder tatsächlichen Einsatz von Kernwaffen ein Angriff auf ein Land als ein Angriff auf alle Länder angesehen wird. Die Teilhabe-Vereinbarungen stellen zweitens unter Beweis, dass die US-Atomstreitkräfte bei einem Krieg Russlands gegen die NATO aufgrund der Rolle vorgeschobener US-Streitkräfte automatisch mit im Spiel wären. Ohne die Teilhabe-Vereinbarungen müssten sich NATO-Mitglieder darauf verlassen, dass eines seiner drei atomar bewaffneten Mitglieder sich nach einem vermutlich langwierigen Entscheidungsprozess bereiterklärte, seine strategischen Streitkräfte in einem Krieg in Europa einzusetzen. Dies würde voraussichtlich die russische Risikobereitschaft erheblich befördern.

Die Methode der kleinen Schritte, die die NATO bei der Entwicklung ihrer Abschreckungsstrategie und -doktrin anwandte, hat zu erheblichen Fortschritten geführt. Aber ist sie erfolgreich gewesen? Hat sie die etwaige Zuversicht von Präsident Putin und seiner engsten Berater, aus einer bewaffneten Konfrontation mit der NATO siegreich hervorzugehen, wirkungsvoll erschüttert - oder täte sie dies in einer Krisenzeit? Die Antwort ist nicht offensichtlich, da die Strategie der NATO nicht am Maßstab dessen überprüft wurde, was eine Theorie des Sieges für erforderlich hält. Es gibt Grund zu Optimismus, da die Anpassungen der NATO an die Situation nach 2014 die konventionelle Abschreckung gegen ein militärisches fait accompli an ihrer Nordflanke deutlich gestärkt haben. Aber es gibt auch Grund zu Pessimismus, da der Erfolg der NATO in grundlegender Weise von der Effektivität abhinge, mit der die Vereinigten Staaten konventionelle Streitkräfte von den Vereinigten Staaten durch feindbedrohte Gebiete in das europäische Einsatzgebiet verlegen könnte. Sie hängt auch davon ab, ob und wie die USA die Eskalation steuern 
Übersicht 2: Elemente einer neuen NATO-Abschreckungsstrategie

\begin{tabular}{|c|c|c|c|}
\hline \multicolumn{2}{|c|}{ NATO Ziele } & Wege & Mittel \\
\hline 1 & $\begin{array}{l}\text { Kampf um den Frieden } \\
\text { gewinnen }\end{array}$ & $\begin{array}{l}\text { - } \quad \text { deutlich das Interesse der transatlantischen } \\
\text { Gemeinschaft an einem freien Europa signali- } \\
\text { sieren } \\
\text { - Erwartungen für Verhalten der NATO in Kriegs- } \\
\text { zeiten deutlich machen }\end{array}$ & $\begin{array}{ll}\text { - } & \text { ein neues Sicherheitskonzept und koordinier- } \\
& \text { tes Signalsenden aus den Hauptstädten } \\
\text { - } \quad \text { Darlegung von NATOs Theorie des Sieges } \\
\text { - } \quad \text { Strategie um Informationskrieg Russlands zu } \\
\text { kontern } \\
\text { - Investitionen in die Fähigkeit, den Gegner mit } \\
\quad \text { Innovationen zu überflügeln }\end{array}$ \\
\hline 2 & $\begin{array}{l}\text { von einer Art. } 2 \\
\text { Bedrohung abschrecken }\end{array}$ & $\begin{array}{l}\text { - } \begin{array}{l}\text { Russland die Erfolge hybrider Kriegsführung } \\
\text { durchkreuzen }\end{array} \\
\text { - } \quad \text { russisches Vertrauen in seine nächsten } \\
\text { Eskalationsschritte erschüttern, indem auf die } \\
\text { Risiken auch nur begrenzter Aktion hingewie- } \\
\text { sen wird }\end{array}$ & $\begin{array}{ll}- & \text { Effektive Heimatverteidigung } \\
- & \text { Vermeidung von Provokationen } \\
- & \text { realistische Fähigkeiten zur Verteidigung und } \\
& \text { Verstärkung und entsprechende Übungen }\end{array}$ \\
\hline 3 & $\begin{array}{l}\text { fait accompli } \\
\text { rückgängig machen }\end{array}$ & $\begin{array}{ll}\text { - } & \text { Russische Invasionsstreitkräfte vertreiben } \\
\text { - } & \text { Vertrauen Russlands in nächste Eskalations- } \\
& \text { schritte erschüttern }\end{array}$ & 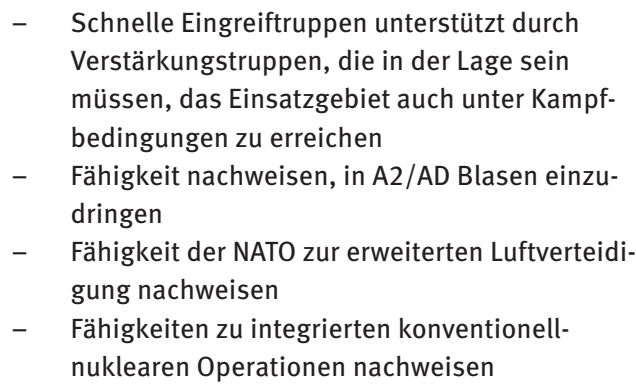 \\
\hline 4 & $\begin{array}{l}\text { Kriegsziele zu er- } \\
\text { reichen trotz Kosten }\end{array}$ & $\begin{array}{ll}\text { - } & \text { Russische Invasionsstreitkräfte zerstören } \\
\text { - } & \text { Krieg ausweiten durch Angriffe auf Logistik } \\
\text { und Infrastruktur in Russland } \\
\text { - } \quad \text { Vertrauen Russlands in nächste Eskalations- } \\
\text { schritte erschüttern }\end{array}$ & 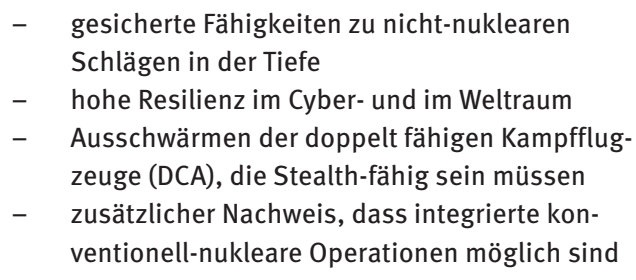 \\
\hline 5 & $\begin{array}{l}\text { von begrenztem } \\
\text { Kernwaffenkrieg in } \\
\text { Europa abschrecken }\end{array}$ & $\begin{array}{ll}\text { - } & \text { Zurschaustellung kollektiver Bereitschaft zur } \\
\text { nuklearen Entschlossenheit } \\
\text { - } \quad \text { Vertrauen Russlands in nächste Eskalations- } \\
\text { schritte erschüttern }\end{array}$ & $\begin{array}{ll}\text { - } & \text { (modernisierte) Arrangements zur nuklearen } \\
\text { - } & \text { Teilhabe } \\
\text { glaubwürdige nukleare Antworten auf } \\
\text { begrenzte Nuklearangriffe auf allen Stufen des } \\
\text { Konflikts, hoher Grad an Bereitschaft } \\
\text { - } \quad \text { Hoher Bereitschaftsgrad der strategischen } \\
\text { Nuklearstreitkräfte der USA (P3) }\end{array}$ \\
\hline 6 & $\begin{array}{l}\text { von begrenzten nicht- } \\
\text { nuklearen Angriffen auf } \\
\text { USA abschrecken }\end{array}$ & $\begin{array}{l}\text { - } \quad \text { Drohungen damit, dass diese Angriffe recht- } \\
\text { zeitig erkannt und abgewehrt werden können } \\
\text { - Vertrauen Russlands in nächste Eskalations- } \\
\text { schritte erschüttern }\end{array}$ & 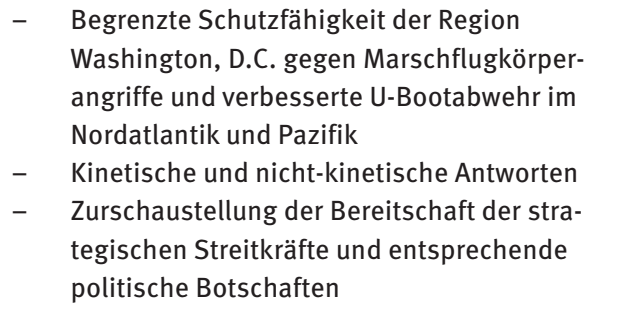 \\
\hline f & $\begin{array}{l}\text { Von Beginn eines } \\
\text { Nuklearwaffenangriffs } \\
\text { auf Russland ab- } \\
\text { schrecken }\end{array}$ & Drohung mit massiver Vergeltung & $\begin{array}{ll}\text { - } & \text { resiliente nukleare Streitkräfte } \\
\text { - } & \text { resiliente Kommandostrukturen (erfordert } \\
& \text { Resilienz in Weltraum und Cyberbereich) } \\
\text { - } & \text { Präsidentielle Stellungnahmen, die Entschlos- } \\
& \text { senheit erkennen lassen }\end{array}$ \\
\hline
\end{tabular}


könnte. Nach Einschätzung der NDS Commission sind sie dazu gegenwärtig kaum in der Lage. ${ }^{28}$

In diesem zwiespältigen Fazit spiegelt sich die Tatsache wider, dass es keine anerkannte Methodik zur Beantwortung der Frage gibt, wie erfolgreiche Abschreckung zu messen sei. Wie die Vereinigten Staaten benötigt auch die NATO eine differenzierte Beurteilungsmethode - eine, die nicht nur das quantitative Kräfteverhältnis, sondern auch die verschiedenen Stärken, Schwächen und Strategien beider Seiten berücksichtigt (net assessment).

\subsection{Ein theoretischer Rahmen für Nordostasien}

Wie das NATO-Bündnis haben sich auch US-Bündnisse mit Japan und Südkorea darauf konzentriert, die Abschreckung an ein verändertes und sich weiterhin veränderndes sicherheitspolitisches Umfeld anzupassen und zu stärken. Diese Allianzen haben das Abschreckungsinstrumentarium in einem weiten Sinne definiert und eine umfassende Anpassungs- und Stärkungsstrategie verfolgt, die sich auf alle militärischen Domänen erstreckt. Eine weitere Ähnlichkeit besteht darin, dass diese Allianzen sich selbst kritisch fragen müssen, ob die hart errungenen Fortschritte der letzten zehn bis zwanzig Jahre ausreichen, um neuen Herausforderungen gerecht zu werden. ${ }^{29}$

Aber die strategische Landschaft in Nordostasien unterscheidet sich in mehreren wichtigen Aspekten von der Landschaft in Europa. Drei grundlegende Unterschiede stechen hervor. Einer ist das Fehlen einer multilateralen Bündnisstruktur. Einerseits macht dies vieles einfacher: Zwei verständigen sich untereinander viel leichter als dreißig. Andererseits bleibt das Verhältnis zwischen Japan und Südkorea aufgrund von Meinungsverschiedenheiten über die Vergangenheit angespannt und wird $\mathrm{zu}$ einem echten Hindernis für eine bessere Koordinierung beim Umgang mit regionalen Bedrohungen. Obgleich Japan sowie Südkorea sich dessen bewusst sind, dass ihre offenen Streitfragen in Krisen- und Kriegszeiten von einem Feind ausgenutzt werden könnten, halten sie an ihren Positionen fest und somit verbleiben hohe Hürden für die Verbesserung der trilateralen Kooperation. Dieser Umstand erlegt einer blauen Theorie des Sieges gewisse Grenzen auf, denn es ist wichtig, Allianzen zusammenzuhalten, während ein Feind sie auseinanderzudividieren versucht.

28 Brauss 2018.

29 Roberts 2013
Ungeachtet ihrer Differenzen hegen sowohl Japan als auch Südkorea zudem Zweifel an der Glaubwürdigkeit der US-Zusage, sie zu verteidigen. Sie befürchten, dass die Vereinigten Staaten sich in Krisen- und Kriegszeiten wegen der damit verbundenen Risiken für US-amerikanisches Territorium von ihrer Verteidigung abkoppeln könnten. Dieser Umstand zeigt, wie wichtig es ist, dass die USA in der Lage sein müssen, das Zwangspotenzial begrenzter Angriffe seitens Chinas zu neutralisieren.

Ein weiter wichtiger Unterschied zwischen Europa und Asien aus der Perspektive von Theorien des Sieges ist die Tatsache, dass es in Ostasien nicht nur ein militärisches Problem gibt. Obgleich sich die NATO vielfältigen sicherheitspolitischen Herausforderungen aus verschiedenen Richtungen gegenübersieht, kommt die größte Herausforderung für ihre Abschreckungsfähigkeiten von Russland. Dies ist offenkundig der einzige gegnerische Staat, der eine Herausforderung für die Beistandsgarantie nach Artikel 5 des NATO-Vertrags darstellen könnte. In Nordostasien dagegen gehen Bedrohungen sowohl von Nordkorea als auch von China aus - Bedrohungen, die von Japan und Südkorea unterschiedlich erlebt werden. Das Bündnis zwischen den USA und Japan muss der doppelten Herausforderung gerecht werden, Japan vor nordkoreanischen Angriffen und vor chinesischen Pressionskampagnen zu schützen, während das US-Bündnis mit Südkorea hauptsächlich darauf ausgerichtet ist, Südkorea vor potenziellen Angriffen Nordkoreas zu schützen. Dies macht es notwendig, die Theorie des Sieges für unterschiedliche Zwecke maßzuschneidern. Die NATO wird sich möglicherweise einer ähnlichen Herausforderung gegenübersehen, wenn ihr Verhältnis zu Iran konfrontativer werden sollte.

Ein dritter, entscheidender Unterschied betrifft die Tatsache, dass Kernwaffen in den militärischen und politischen Strategien Chinas eine viel geringere Rolle spielen als in den Strategien Russlands. Dies soll nicht heißen, dass ihre Rolle unbedeutend ist; im Gegenteil, China misst seinem nuklearen Abschreckungspotenzial einen hohen Stellenwert bei, wie seine beträchtlichen Investitionen in die Modernisierung und Diversifizierung dieser Streitmacht in den letzten Jahrzehnten bezeugen. Aber während Russland seine Atomstreitkräfte in den Mittelpunkt seiner Strategie stellt und sich auf sämtlichen Ebenen des Kampfes auf eine nukleare Kriegsführung vorbereitet hat, hält China seine Atomstreitkräfte im Hintergrund, bleibt bei einer Strategie des Ersteinsatzverzichts und rüstet sich dafür, Konflikte mit konventionellen Mitteln zu kontrollieren und für sich zu entscheiden. Dies stellt andere Anforderungen an die blaue Theorie des Sieges, als es bei der hohen konventionell-nuklearen Integration der Fall ist, die die russische Strategie kennzeichnet. 
Daher sind US-Verteidigungsplaner gezwungen, für diese Region eine ganze Reihe von Szenarien durchzuspielen. Viele davon liegen in der Grauzone und werden daher im nächsten Kapitel behandelt. Einige umfassen bewaffnete Konfrontationen mit Nordkorea. Aber hier liegt der Schwerpunkt auf Kriegen zwischen Großmächten, und das mit Abstand am stärksten beunruhigende Ereignis wäre in dieser Hinsicht ein potenzieller Krieg zwischen China und den Vereinigten Staaten um Taiwan. Dies sticht als ein Sonderfall heraus, weil aus Sicht Chinas dabei Fragen der nationalen Souveränität auf dem Spiel stünden, während es aus US-amerikanischer Sicht um Werte geht, die für die Nation und für die internationale Ordnung von großer Bedeutung sind.

In diesem Zusammenhang haben Verteidigungsexperten in den letzten Jahren unterschiedliche Prämissen diskutiert, auf deren Grundlage sich eine blaue Theorie des Sieges erstellen ließe:

1. Würde Taiwan ohne Provokation angegriffen, täten die Vereinigten Staaten mit Entschlossenheit das Notwendige, um dem Staat militärischen Beistand zu leisten. Dazu gehörten auch Zwangsandrohung und begrenzte Demonstrationen der Stärke. Der Versuchung Chinas, einen militärischen fait accompli zu schaffen, könnte man dadurch entgegenwirken, dass man Taiwans Fähigkeit stärkte, den Streitkräften der VR China im Falle eines Angriffs hohe Kosten aufzuerlegen.

2. Jeder Versuch, die Vereinigten Staaten durch Androhung oder Durchführung begrenzter konventioneller Raketenangriffe auf US-Streitkräfte, US-Verbündete und womöglich US-Territorium selbst von der Verteidigung Taiwans abzukoppeln, wird nur das Gegenteil des Gewünschten bewirken.

3. Peking hat viele wirtschaftliche, politische und militärische Gründe, einen Konflikt um Taiwan, der für China schlecht läuft, nicht zu eskalieren. Aber wenn es dies trotzdem täte (vertikal oder horizontal), dann könnte seine Führung durch die Folgen massiver militärischer Schläge der USA, die dabei auf breitgefächerte kinetische und nicht kinetische Angriffsfähigkeiten zurückgriffen, zu der Einsicht gebracht werden, dass sie sich verkalkuliert hat. Die Vereinigten Staaten, Taiwan und vielleicht auch US-Verbündete in der Region könnten den erwarteten Nutzen begrenzter kinetischer und nicht kinetischer Angriffe auf sie auch durch Raketenabwehrsysteme und Resilienz im Cyberspace und im Weltraum verringern.

4. Es ist äußerst unwahrscheinlich, dass China in diesem Szenario einen nuklearen Erstschlag gegen amerikanische Streitkräfte in der Region oder gar gegen das
US-Territorium führen würde. Dies würde auch dann nicht der Fall sein, wenn China eine Niederlage in einem Konflikt um Taiwan rückgängig machen wollte. China könnte eine solche Niederlage verkraften auf der Basis des Arguments, dass Taiwan nicht verschwinden werde und Chinas Position sich langfristig nur verbessern könne.

5. Für die Vereinigten Staaten steht in einem Krieg mit China wegen Taiwan genauso viel auf dem Spiel wie für China. Die Werte Amerikas wären bedroht. Außerdem untergrübe es US-Sicherheitsgarantien weltweit, wenn die USA nicht entschieden reagierten.

Für das US-Militär ist die Verteidigung Taiwans ein andauernder Auftrag. Für China dagegen haben Vorbereitungen für eine militärische Lösung des langjährigen Konflikts eine hohe nationale Priorität. Allerdings konzentriert sich die chinesische Militärplanung nicht allein auf einen möglichen Taiwan-Konflikt. Die Erwartung einer möglichen bewaffneten Konfrontation mit den Vereinigten Staaten ist ein Schlüsselfaktor bei der Modernisierung des chinesischen Militärs. ${ }^{30}$ Dies bedeutet, dass die US-amerikanische Theorie des Sieges in einem solchen Eventualfall womöglich einer ernsthaften Bewährungsprobe unterzogen würde und daher robust sein müsste.

Wenn es darum geht, Fortschritte bei der Bewältigung dieser Herausforderungen zu beurteilen, sind die Vereinigten Staaten und ihre ostasiatischen Verbündeten abermals mit der Schwierigkeit konfrontiert, dass sie über keine geeignete differenzierte Methode der Einschätzungen der verschiedenen Optionen (strategic net assessment) verfügen.

\subsection{Aufgabe von Blau: Vorbereitungen für zwei Operationsgebiete treffen}

Die blaue Rahmentheorie des Sieges muss auch auf die Tatsachen zugeschnitten werden, dass erstens nur die Vereinigten Staaten die Sicherheit von Staaten in zahlreichen Regionen garantieren und dass zweitens der Erfolg in einem Krieg gegen eine Großmacht viele der militärischen Ressourcen beansprucht, die gegenwärtig für andere Zwecke global disloziert sind. Die NDS Commission gelangte zu dem Schluss, diese Ressourcen könnten nur dann effektiv genutzt werden, wenn sie aus anderen Regionen und von Verbündeten abgezogen würden. ${ }^{31}$ Einem

30 Scobell/Kamphausen/Lai 2009.

31 Mitre 2019. 
Operationsgebiet werden also Ressourcen zugunsten eines anderen entzogen. Dies ist besonders beunruhigend $\mathrm{zu}$ einer Zeit enger strategischer Kooperation zwischen den Präsidenten Putin und Xi, da eine opportunistische Aggression in einer Region mit einer opportunistischen Aggression in einer anderen Region koordiniert werden könnte, mit der Erwartung, dass dadurch die USA in beiden eine Niederlage erlitten.

Wenn die Vereinigten Staaten dagegen wirksam Abhilfe schaffen wollten, müssten sie schwierige Entscheidungen treffen. Eine Abhilfe bestünde darin, die Rolle nuklearer Abschreckung anzuheben, um konventionelle Defizite wettzumachen. Die Vereinigten Staaten mussten seit Beginn des Kalten Krieges einen solchen Schritt nicht mehr in Erwägung ziehen, und jahrzehntelang gab es einen breiten Konsens zwischen Demokraten und Republikanern, die Rolle von Kernwaffen zu reduzieren. Wollte man diese jetzt wieder aufwerten, bedürfte es hierzu einer neuen deklaratorischen Nuklearpolitik, die in Anbetracht ihrer besonderen Zielsetzungen in Abstimmung mit US-Verbündeten entwickelt werden müsste. Eine nukleare Aufwertung erforderte wahrscheinlich auch Änderungen der Einsatzdoktrin: Einige der zuvor aus Europa abgezogenen Ressourcen könnten wieder erneut dorthin verlegt werden oder solche Ressourcen könnten in Friedenszeiten dauerhaft in oder in der Nähe von Ostasien stationiert werden. Solche Anpassungen der Doktrin werden einhergehen mit heiklen neuen Fragen über das nötige Maß der Koordinierung der Entscheidungsfindung zwischen den Vereinigten Staaten und ihren Verbündeten in Bezug darauf, ob, wann und wie US-Kernwaffen eingesetzt werden sollten.

Eine andere Form der Abhilfe bestünde darin, dass die Alliierten der USA sich stärker einbrächten und erhöhte militärische Beiträge leisteten. In den letzten zwanzig Jahren haben US-Regierungen im Allgemeinen die Beiträge von Alliierten zu regionalen Abschreckungsarchitekturen als förderlich für die Abschreckung und als nützlich für die Lastenteilung begrüßt. Aber zugleich wehrten sie sich gegen Beschneidungen ihres Handlungsspielraums, die mit der Abhängigkeit von diesen Alliierten verbunden sind. Und sie hüteten sich auch davor, die Angriffsfähigkeiten von Verbündeten deutlich zu verbessern, aus Sorge, diese könnten in Krisenzeiten selbstständig agieren. Eine glaubwürdige Theorie des Sieges in dem vernachlässigten zweiten Operationsgebiet macht es aber erforderlich, dass sich die Vereinigten Staaten stärker auf Verteidigungs- und auch auf die vorhandenen Abschreckungsfähigkeiten von Alliierten stützen und dass sie bereitwilliger ein glaubhaftes nukleares Abschreckungsmittel für dieses konkrete Problem zur Verfügung stellen.

\subsection{Einschränkende Anmerkungen}

Wie bei der roten Theorie des Sieges in Krisen und Kriegen gibt es auch bei der blauen Theorie Aspekte, die sich vielleicht nicht als zutreffend erweisen, wenn sie in Krisen und Kriegen einer praktischen Bewährungsprobe unterzogen werden. Ein starkes konventionelles Abschreckungsmittel kann zu einem lohnenden Ziel für einen Angreifer werden (wie die in Pearl Harbor stationierten US-Seestreitkräfte im Jahr 1941). ${ }^{32}$ Beteuerungen der USA, einen Angriff auf einen Verbündeten als einen Angriff auf sich selbst zu betrachten, mögen Gegnern der USA nicht als glaubhaft erscheinen, mit der Folge, dass sie vielleicht die Probe aufs Exempel suchen. In einem sich ausweitenden Krieg sind US-Verbündete vielleicht nicht hinreichend siegesgewiss, um weiterzukämpfen. Die Verantwortlichen in Washington kommen vielleicht zu dem Schluss, dass für die USA bei der Verteidigung einer regionalen Ordnung tatsächlich weniger auf dem Spiel stünde als für den Herausforderer, was sie dazu veranlassen könnte, wichtige Interessen von Verbündeten zu opfern. Die politischen Entscheidungsträger auf Seiten der Gegner haben möglicherweise verhältnismäßige Gegenschläge der Vereinigten Staaten und ihrer Verbündeten von vornherein miteinkalkuliert und stecken sie ohne nennenswerten strategischen Effekt weg. Die Vereinigten Staaten und ihre Alliierten müssten dann zusätzliche Eskalationsschritte erwägen. Eine verspätete Reaktion auf nukleare Aggressionen von Rot könnte bei dessen politischen und militärischen Führern als mangelnde nukleare Entschlossenheit interpretiert werden und diese möglicherweise $\mathrm{zu}$ weiteren nuklearen Angriffen ermutigen. US-Militäraktionen könnten Entscheidungsträger des Gegners erzürnen, anstatt sie zur Vernunft zu bringen, und sie könnten den Schluss ziehen, dass weitere derartige Aktionen die nationale Integrität und ihre politische Kontrolle bedrohten, sodass sie sich womöglich für eine weitere Eskalation entscheiden. Die in diesem Stadium angebotenen „Ausstiegsoptionen“ erscheinen einem verwundeten und wütenden - aber noch immer mächtigen Gegner möglicherweise als unannehmbar. Angesichts dieser Möglichkeiten fällt den US-Verteidigungsplanern die Aufgabe $\mathrm{zu}$, Hypothesen $\mathrm{zu}$ testen, Annahmen $\mathrm{zu}$ klären und Wunschdenken und Selbstüberschätzung von wohldurchdachten Analysen zu trennen. Dies erhöht auch die Dringlichkeit einer substanziellen Zusammenarbeit mit US-Verbündeten, um sicherzustellen, dass das neue Strategiekonzept kohärent ist und Lernprozesse beschleunigt werden.

32 Schelling 1960b. 
Auch wenn reguläre Streitkräfte normalerweise in einem regionalen Kontext zusammenstoßen und auch wenn das hauptsächliche Ziel von Rot die Kompromittierung der US-gestützten regionalen Sicherheitsordnung wäre, so hätten solche Kriege wahrscheinlich doch auch erhebliche transregionale Auswirkungen. Rot könnte es als nützlich erachten, US-Streitkräfte in anderen Regionen anzugreifen, um ihre Verlegung in das relevante Einsatzgebiet zu hemmen. Rot könnte es auch als notwendig erachten, kritische Infrastruktur in den Vereinigten Staaten, die für die schnelle Verlegung von US-Streitkräften unverzichtbar ist, zu einem relativ frühen Zeitpunkt in einem Konflikt anzugreifen, um die Verlegung zu bremsen. Und Rot könnte es auch als notwendig erachten, in den späteren Phasen eines Konflikts US-Territorium selbst anzugreifen, um die USA dazu zu bewegen, den Krieg zu beenden, bevor Rot den nächsten größeren Eskalationsschritt machte und einen breiter angelegten Angriff auf US-Territorium startete. Diese Angriffe zielten darauf ab, die USA „zur Vernunft zu bringen,“ nicht darauf, sie zu erzürnen. Vermutlich wären sie begrenzt; sie sollten den Amerikanern vor Augen führen, dass ihnen Schlimmeres drohen könne.

Die Analyse der Theorien des Sieges von Rot und Blau verdeutlicht auch die potenzielle Eskalationsdynamik in einem solchen Krieg. Genauer gesagt hat sie die übliche Vorstellung etwas komplexer gemacht. Die übliche Vorstellung ist die einer Glockenkurve, die das Ausmaß der Konflikteskalation als eine Funktion der Entscheidung jedes Akteurs darstellt, an kritischen Punkten zu eskalieren oder zu deeskalieren. Tatsächlich besteht ein solches Modell aus mindestens drei Kurven: der roten Kurve, der blauen Kurve und der Kurve der US-Verbündeten. Jeder Kriegsteilnehmer kämpft auf seinem eigenen Niveau und auf seine eigene Weise; der eine muss dem anderen bei der Eskalation oder Deeskalation nicht folgen. Jeder Akteur steht vor einer komplexen Entscheidung zwischen Eskalation, Deeskalation und Fortsetzung des Kampfes mit bisheriger Intensität. Außerdem müssen bei jedem Eskalationsschritt Entscheidungen darüber getroffen werden, welche Kosten, aber auch welche Risiken man auferlegen will - und umgekehrt, welche Risiken man als Signal der Entschlossenheit akzeptiert.

Die Tatsache, dass der moderne Krieg in mehreren Domänen ausgetragen wird, macht das Ganze noch komplexer, da die Konfliktparteien heute über zusätzliche Mittel verfügen, mit denen sie der anderen Seite Kosten und Risiken auferlegen können. Aber sie erhöht auch das Risiko ungewollter Konsequenzen ganz erheblich. Schon Hermann Kahn hat die Komplexität einer potenziellen nuklearen Eskalation mit seinem aus 44 Sprossen beste- henden Modell der nuklearen Eskalationsleiter umfassend demonstriert. ${ }^{33}$ Die Mehr-Domänen-Herausforderung hat einige dazu veranlasst, stattdessen ein Eskalationsgitter vorzuschlagen. ${ }^{34}$ Aber die Herausforderung lässt sich möglicherweise leichter bewältigen, als es den Anschein hat. Erinnern wir uns, dass parallel zu Kahns Sprossen eine Achse mit einer viel kleineren Zahl wichtiger Schwellen verläuft: Krisenintensivierung, regionaler Krieg, Angriffe auf „zentrale Sanktuarien“ und allgemeiner Krieg. Diese sind auch im zeitgenössischen Kontext nach wie vor relevant. Anders als die 44 Sprossen sind dies die Eskalationsschwellen, die im Hinblick auf ihre Auswirkungen auf das gegnerische Nutzen-, Kosten- und Risikokalkül bei einer Fortsetzung des Krieges am meisten entscheidend sind..$^{35}$

Diese Rot-Blau-Analyse liefert noch einen weiteren letzten Aufschluss über das Problem der Eskalation. Dies sind die Ungewissheit und das Risiko, die mit der Überzeugung verbunden sind, es sei möglich, einen Feind durch Verabreichung genau der „verordneten Dosis“ Schmerzen „zur Vernunft und nicht in Rage zu bringen.“ Dies mag sich als unmöglich erweisen. Es dürfte besonders aussichtslos in Krisen sein, in denen für beide Seiten ungefähr gleich viel auf dem Spiel steht. Ein entsprechendes Vorgehen könnte daher katastrophale Folgen haben.

\section{Die Grauzone}

Eine Theorie des Sieges, die Konflikte in der Grauzone nicht einbezieht, ist unvollständig. Aber was ist die Grauzone? Was ist das besondere Gepräge von Konflikten, die sich dort abspielen? Was bedeutet Sieg in der Grauzone? Frank Hoffman hat zu Recht darauf hingewiesen, dass „Grauzonen-Konflikte nach wie vor sehr weit und vage definiert" seien. ${ }^{36}$ Jede neue diesbezügliche Erfahrung scheint die Aufmerksamkeit des Westens auf sich gezogen zu haben: Russlands „grüne Männchen“ in der Ukraine, die maritime Miliz Chinas, die politische Kriegsführung Russlands gegen westliche Demokratien, Chinas Desinformationsstrategien und die „aktiven Maßnahmen“ Russlands gegen westliche Bürger und anderes mehr.

Es läge nahe, in den Schriften russischer und chinesischer Experten und Amtsträger nach einer Definition von Grauzonen-Konflikten zu suchen. Aber es gibt von ihnen nur relativ wenige Publikationen zu diesem Thema.

33 Kahn 1965.

34 Lindsay/Gartzke 2019.

35 Talmadge 2019.

36 Hoffman 2019. 
Sie verwenden nicht den Terminus „Grauzone.“ Dies mag mit der Tatsache zusammenhängen, dass viele Grauzonen-Aktivitäten illegal sind. Es könnte auch sein, dass diese Aktivitäten eher spontan und opportunistisch als im wirklichen Sinne „strategisch“ sind. Vielleicht gibt es keine systematische Theoriebildung über den Sieg in der Grauzone. Oder diese beginnt gerade erst. Oscar Jonsson vertritt die Auffassung, dass „die Erweiterung des russischen Verständnisses von Kriegsführung ein langfristiger, evolutionärer Prozess gewesen ist.“" ${ }^{37}$ Es kann aber auch sein, dass entsprechende Konzepte der Geheimhaltung unterliegen. Chinesische Militärexperten sind einer Definition recht nahegekommen - mit dem Terminus „Quasi-Krieg.“ Dieser sei ein „mittlerer Punkt“ auf dem Konfliktspektrum. Das Militär sei hier beteiligt, aber ein Krieg sei noch nicht ausgebrochen. Es sei ein Zustand mit Merkmalen von beiden - Krieg und Frieden..$^{38}$ Russische Experten konzentrieren sich darauf, die Grenze zwischen Frieden und Krieg zu verwischen. ${ }^{39}$

Für die Zwecke dieser Analyse definieren wir Grauzone als den Abschnitt des Konfliktspektrums, in dem es keine bewaffneten Feindseligkeiten gibt. In gewissem Sinne ist es eine Friedenszone, weil noch kein richtiger Krieg ausgebrochen ist. Andererseits ist es kein Friedenszustand, da der Konflikt bereits im Gange ist und auch unterschiedliche militärische Aktionen stattfinden. In gewissem Sinne greift der Begriff Grauzone eine alte Idee auf: das Streben nach Vorteilen, ohne sich den Kosten und Risiken eines Krieges auszusetzen. So schrieb etwa Edward H. Carr im Jahr 1939: „Das allgemeine Interesse am Frieden verschleiert die Tatsache, dass einige Nationen den Status quo aufrechterhalten wollen, ohne dafür kämpfen zu müssen, während andere den Status quo verändern wollen, ohne dafür kämpfen zu müssen.“40 Aber der Begriff erfasst auch etwas Neues: die asymmetrischen Strategien gegnerischer Großmächte. Erinnern wir uns an Peter Roberts Feststellung, wonach die Gegner des Westens „die Kriegskunst neu konzipiert und den Begriff des Konflikts neu definiert haben. ${ }^{“ 41}$ Dies zeigt sich nirgendwo deutlicher als in ihren Strategien für die Grauzone. Konflikte in der Grauzone sind kein Nebenaspekt der neuen strategischen Herausforderung; sie bilden deren Kern.

37 Jonsson 2019, 7.

38 Peng/Yao 2015. See also Erickson/Martinson 2019.

39 Gerasimov, World on the Brink of War, op. cit.

40 Carr 1939, 52-53.

41 Roberts 2019.

\subsection{Rote Konfliktstrategien in der Grauzone}

Der Katalog russischer Aktivitäten in der Grauzone ist ebenso eindrucksvoll wie beunruhigend. Dazu gehören zum Beispiel aktive Maßnahmen gegen einflussreiche Ziele, gezielte Tötungen sogenannter Staatsfeinde, Einmischungen in westliche Wahlprozesse, Informationskonfrontationsstrategien, Maßnahmen zum Einfrieren von Konflikten in der Peripherie und das Bestreben, in US-Einflussregionen (erneut) Einfluss zu nehmen. Solche Aktivitäten stehen voll und ganz in Einklang mit den primären Zielen der Grand Strategy Russlands, wie sie Präsident Putin definiert hat.

Auch der Katalog chinesischer Aktivitäten ist eindrucksvoll und beunruhigend. Dazu gehören u.a. die entschlossene Durchsetzung eigener Interessen in der maritimen Domäne, der Einsatz maritimer Milizen, Informationskonfrontationsstrategien und die gezielte Schaffung wirtschaftlicher Abhängigkeitsverhältnisse mit Nachbarn in Eurasien. Derartige Aktivitäten stehen voll und ganz in Einklang mit den primären Zielen der Grand Strategy, wie Präsident Xi sie definiert hat.

Was könnte „Sieg“ in der Grauzone für Moskau und Peking bedeuten? Die Antwort lautet, dass „Sieg“ hier im Wesentlichen das Gleiche bedeutet wie „Sieg im Krieg“: die Realisierung hochrangiger strategischer Ziele, die von der nationalen Führung festgelegt worden sind. Diese Ziele laufen darauf hinaus, in revisionistischer Absicht die Weltordnung von Grund auf neu zu gestalten und dabei in dem Konflikt die Oberhand zu gewinnen, der ihnen ihrer Auffassung nach von den Vereinigten Staaten aufgenötigt wurde. „Sieg“ bedeutet in diesem Sinne nicht so sehr, in der Grauzone zu gewinnen, als vielmehr durch die Grauzone zu gewinnen. Das heißt, es geht darum, geringfügige Vorteile, die sukzessive auf der operativen Ebene der Strategie errungen werden, in strategische Vorteile in ihrem langfristigen Konflikt mit den Vereinigten Staaten über die Ausgestaltung der internationalen Ordnung umzumünzen.

Wie lassen sich ihrer Auffassung nach diese Ziele erreichen? Erinnern wir uns daran, dass ihre Theorien des Sieges in Krisen und Kriegen auf der Idee beruhen, die Vereinigten Staaten und ihre Verbündeten an zentrale Entscheidungspunkte zu führen, an denen sie die Wahl zwischen „Pest“ oder „Cholera“ haben: zwischen Eskalation und Beschwichtigung im Kontext regionaler Kriege. In der Grauzone dagegen ist die Strategie von Rot auf das Gegenteil ausgerichtet. Rot will sicherstellen, dass die Vereinigten Staaten und ihre Verbündeten nie vor die Entscheidung gestellt werden, ein Interesse zu verteidigen. Entscheidungspunkte werden vermieden, nicht geschaffen. Dies 
ist die sogenannte Boil-the-Frog-Strategie: Man erhitzt das Wasser langsam, und der Frosch bemerkt die Gefahr erst, wenn er schon gelähmt ist. Russland und China versuchen, die internationalen Ordnungen in ihrem regionalen Umfeld langsam umzugestalten, ohne eine scharfe Gegenreaktion hervorzurufen. Russland zum Beispiel will das politische System in den USA lähmen, während es gleichzeitig auf Sanktionserleichterungen drängt. China will seine Ansprüche auf unbewohnte Riffs zur Geltung bringen, indem es Stützpunkte darauf errichtet, ohne eine gemeinsame japanisch-amerikanische Militäraktion auszulösen, um diese Ansprüche zurückzuweisen. Selbstverständlich müssen die Führungen in Russland und China eine Vorstellung von einem „Kulminationspunkt“ haben, an dem sich die Vereinigten Staaten und ihre Verbündeten politisch mit der neu gestalteten Ordnung abfinden. Aber in der Grauzone streben sie eine stillschweigende Inkaufnahme und keine dramatische Kapitulation an. Sie wollen ihren Feind kampflos bezwingen.

Ein weiteres zentrales Merkmal der roten Theorie des Sieges in der Grauzone ist der hohe Stellenwert der Auferlegung von Risiken. Dadurch, dass sie durch Aktionen in der Grauzone die Risiken für die Vereinigten Staaten und ihre Verbündeten erhöhen, anstatt ihnen Kosten aufzuerlegen, wollen die Führer in Russland und in China die Entschlossenheit der Vereinigten Staaten und ihrer Verbündeten in einem langsamen Prozess der Gewöhnung untergraben. Dabei nutzen sie internationale Krisen aus. Westliche Staats- und Regierungschefs bemühen sich im Allgemeinen darum, Krisen so schnell wie möglich $\mathrm{zu}$ deeskalieren und beizulegen. Russische und chinesische Krisenmanager hingegen sind in der Regel bestrebt, Krisen in die Länge $\mathrm{zu}$ ziehen und die Eskalationsschraube bald anzuziehen, bald zu lockern, um die Vereinigten Staaten und ihre Verbündeten psychologisch unter Druck zu setzen. ${ }^{42}$ Westliche Staaten setzen dabei gerne „rote Linien.“ Für Rot können derartige „rote Linien“ aber nützlich sein, die von Blau gezogen werden. Diese verringern Ungewissheit und Risiken und stecken Handlungsspielraum deutlich ab. Sie sind auch nützlich, um einzuschätzen, wo man hin und wieder eine Linie übertreten kann. Diese Theorie des Sieges hat die gleiche Schwachstelle wie die Theorie des Sieges in Krisen und Kriegen. Sie scheint davon auszugehen, dass der Frosch die Gefahr nicht bemerkt und dem ihm zugedachten Schicksal daher nicht entrinnen kann.

42 Swaine/Zhang/Cohen 2006.

\subsection{Elemente einer blauen Theorie des Sieges in der Grauzone}

Im Zuge ihrer Bemühungen, die Grauzone und die besondere Eigenart der Konflikte in ihr zu definieren, haben Experten und Entscheidungsträger in den Vereinigten Staaten auch darüber diskutiert, wie man in geeigneter Weise darauf reagieren sollte. Es wurden verschiedene Modelle entwickelt. Eines ist ad hoc und reaktiv. Es geht davon aus, dass es einer mehrgleisigen Reaktion auf die mehrgleisigen Grauzonen-Herausforderungen durch Russland und China bedarf. Sie umfasst zum Beispiel Aktionen, die darauf gerichtet sind, Versuche der politischen und militärischen Zwangsausübung zu vereiteln, illegale Handlungen aufzuspüren und aufzudecken, elektronische und anderweitige Störungen in politischen Systemen des Westens zu unterbinden. In Anbetracht der großen Vielfalt von „Mitteln,“ die heute eingesetzt werden, führt dies zu einer umfangreichen und komplexen politischen Agenda, welche sämtliche Institutionen und Personen einbezieht, die für die Abwehr von Rot in der Grauzone verantwortlich sind. Dies ist die gegenwärtige Vorgehensweise der USA.

Ein weiteres Modell ist stärker strategisch ausgerichtet. Hier ist das beste Exempel das „affirmative Programm ... von Methoden unterhalb der Kriegsschwelle ... zur Abwehr sowjetischer Bedrohungen“ in dem unter der Bezeichnung „NSC 68“ bekannten Dokument aus dem Jahr 1950, in welchem die Eindämmungsstrategie des Kalten Krieges dargelegt wurde..$^{43}$ Das „affirmative Programm“ legte spezifische strategische Ziele und die verschiedenen Vorgehensweisen zu ihrer Realisierung fest. Die Auswahl der konkreten Mittel überließ es den verschiedenen Ministerien. Die daraus abgeleitete Theorie des Sieges besagt, dass eine sorgfältige Balance von Entschlossenheit und Zurückhaltung seitens des Westens die Sowjetunion eindämmen und ihren Zusammenbruch fördern werde. Von diesen Modellen dürfte das NSC-68-Modell dasjenige sei, das sich für die Vereinigten Staaten am besten eignet.

Eine blaue Theorie des Sieges setzt voraus, dass Sieg sinnvoll und möglich ist. Viele bezweifeln, dass dies in der Grauzone der Fall sei. Sie sind der Auffassung, die Vereinigten Staaten und ihre Verbündeten befänden sich in ihrer Rolle als Status-quo-Mächte zwangsläufig in einem reaktiven Modus. Hier ist es hilfreich, sich daran zu erinnern, was auf dem Spiel steht. Die Führer in Russland und

43 NSC-68. A Report to the National Security Council on United States Objectives and Programs for National Security (April 14, 1950), 62-63; https://digital-commons.usnwc.edu/cgi/viewcontent.cgi? article $=5901 \&$ context $=$ nwc-review . 
China wollen die regionalen und globalen Ordnungen neugestalten. Wir sollten verstehen, dass der Zusammenbruch der NATO in Europa, des europäischen Projekts allgemein und von US-Bündnissen in Ostasien zum einen zu einer deutlichen Zunahme der Wahrscheinlichkeit von Kriegen und zum zweiten zum Verlust von Souveränität und strategischer Unabhängigkeit demokratischer Staaten in Europa und Ostasien führen wird. In der Grauzone steht viel mehr auf dem Spiel als eine vermeintlich heruntergekommene internationale Ordnung und ein paar umstrittene Felsriffe in den Meeren in Chinas Nachbarschaft.

Die Idee des „Sieges“ ist daher ein sinnvolles Konzept auf strategischer Ebene für Konflikte in der Grauzone. Es bedeutet, dass man Entscheidungsträger in Moskau, Peking, Pjöngjang und Teheran an einen „Kulminationspunkt" bringt, an dem sie die internationale Ordnung oder friedliche politische Mittel zu ihrer Veränderung akzeptieren, anstatt sie weiterhin zu bekämpfen. Sie würden sich entscheiden, die bestehenden regionalen und globalen Ordnungen hinzunehmen und sie würden aufhören, westliche politische und Sicherheitsinteressen zu untergraben. Sie müssten ihre Wettbewerbsambitionen auf legitime wirtschaftliche oder politische Felder richten. Die Rivalität würde nicht aufhören, Konflikte dagegen schon.

Wenn man dieses Ziel erreichen will, dann müsste man die entsprechenden politischen Führer und Entscheidungsträger davon überzeugen, dass die Kosten und Risiken ihrer gegenwärtigen Handlungsweisen in einer Weise steigen werden, die für sie unannehmbare Kosten verursachen werden. Gleichzeitig muss ihnen gegenüber signalisiert werden, dass ihnen alternative Handlungsweisen offenstehen, die geringere Kosten und Risiken sowie eine Reihe wirtschaftlicher, politischer und Sicherheitsvorteile versprechen. Sie müssten auch darauf vertrauen können, dass diese Vorteile von Dauer sind. Die Theorie erfordert zudem, dass sie selbst als instabile Regime der Zusicherung der Vereinigten Staaten und ihrer Verbündeten Glauben schenkten, dass ihre Interessen auch dann respektiert würden, wenn sie diese nicht mit militärischen Mitteln vorantrieben.

Eine mindere Form von Sieg, wenn auch trotzdem ein Sieg, wäre es, wenn ihre Bemühungen, die darauf abzielen, mit militärischen und wirtschaftlichen Drohungen die USA und ihre Verbündeten zu etwas zu zwingen, erfolgreich vereitelt würden. Auch wenn die politischen Führer von Rot vielleicht nicht dazu gebracht werden können, ihre Ziele und Mittel aufzugeben, können sie politisch bedeutungslos gemacht werden, wenn die Drohungen unglaubwürdig erscheinen. Die wichtigsten unterstützenden Elemente dieser blauen Theorie des Sieges in der Grauzone sind die folgenden:
- Ein umfassender und koordinierter Ansatz von Blau zur Stärkung von Verteidigungsfähigkeiten und Resilienz führt dazu, dass Erfolge von Rot vereitelt und dessen Selbstvertrauen untergraben wird.

- Blau kann im Lauf der Zeit mit robusteren Wettbewerbsstrategien in Wissenschaft, Technik und anderen Sektoren das Zwangspotenzial der militärischen Fähigkeiten von Rot untergraben.

- Die Vereinigten Staaten und ihre Verbündeten können sich aktiv darum bemühen, die bestehenden regionalen Sicherheitsordnungen aufrechtzuerhalten und diese Ordnungen selektiv zu erweitern und zu vertiefen. Mit einem Bekenntnis zu kollektiver Sicherheit, Freihandel und gemeinsamen Werten können sie das Selbstvertrauen von Rot ebenfalls kontinuierlich untergraben.

- Die Vereinigten Staaten und ihre Verbündeten können anderen wichtigen Mächten (Indien, Brasilien, Indonesien) eine dauerhafte Kooperation von bedeutendem Umfang anbieten. Eine solche Kooperation trüge dazu bei, die Debatten innerhalb der politischen Entscheidungsgremien von Rot zu beeinflussen.

- Die Vereinigten Staaten und ihre Verbündeten können das Selbstvertrauen von Rot erschüttern, indem sie jene Voraussetzung schaffen, die es dem Westen erlaubt, Krisen und Kriege erfolgreich zu bestehen. Dazu ist es erforderlich, Theorien des Sieges für die blaue Seite zu erarbeiten und für Abschreckung und Verteidigung maßgeschneiderte Fähigkeiten zu entwickeln.

- Die Vereinigten Staaten und ihre Verbündeten können einen Regimewechsel in Moskau und Peking abwarten. Der Abgang der gegenwärtigen Regime wird ein Zeitfenster eröffnen, in dem die politischen Beziehungen erneuert und verbessert werden können.

\section{Fazit}

Der Westen sieht sich einer neuen strategischen Herausforderung durch Russland und China gegenüber, die umfangreiche Vorbereitungen für regionale Kriege mit den Vereinigten Staaten und ihren Verbündeten treffen. Diese Herausforderung ist Folge eines breiten, mehrdimensionalen Konfliktes, den beide Länder mit den Vereinigten Staaten austragen. Dieser hat militärische, politische, wirtschaftliche und ideologische Aspekte und zielt darauf ab, die regionalen Ordnungen in ihrer Nachbarschaft, aber auch die internationale Ordnung neu zu gestalten. So wie ihn die Regierenden in Russland und China defi- 
niert haben, ist der Konflikt ein Nullsummenspiel. In ihrer Sichtweise erstreckt sich dieser Konflikt über Friedens-, Krisen- und Kriegszeiten. Daher haben sie Strategien entwickelt, um ihre Interessen auf der ganzen Länge dieses Kontinuums zu fördern. Der strategische Charakter dieses Konflikts rührt zum Teil daher, dass Kernwaffen und andere wirksame militärische oder hybride Fähigkeiten eine wichtige Rolle spielen. Auf dem Spiel stehen aber auch die regionalen Sicherheitsordnungen in Europa und Ostasien, eine Reihe globaler Institutionen und Normen und auch die Reputation der Vereinigten Staaten als ein verlässlicher Garant der Sicherheit anderer Länder.

Damit westliche Strategien diese neuen Herausforderungen erfolgreich meistern, bedarf es einer westlichen (blauen) Theorie des Sieges. Da der Begriff nicht allgemein eingeführt ist, muss er präzise definiert werden. Ganz einfach ausgedrückt besteht eine Theorie des Sieges aus einer Reihe plausibler Grundsätze zur Bezwingung eines Feindes. Genauer gesagt besteht sie aus einer Reihe von Aussagen darüber, wie und warum das Verhalten einer Konfliktpartei in einer gewünschten Art und Weise beeinflusst werden kann. Eine Theorie des Sieges erklärt, wie man einen Feind an einen „Kulminationspunkt“ bringt, an dem er sich entschließt, die Kosten und Risiken der Fortsetzung des Konflikts nicht auf sich zu nehmen und sich dadurch, dass er den Konflikt beendet, mit den Präferenzen des ersten Akteurs abfindet. Eine Theorie des Sieges verlangt ein robustes Konzept des Sieges, das den Kontext sowie die verschiedenen Konfliktphasen einschließlich der Nachkriegsphase berücksichtigen muss.

Eine blaue Theorie des Sieges ist nach wie vor nicht hinlänglich ausgearbeitet. Sie lässt sich in einem dreistufigen Prozess weiterentwickeln: Erstens, Blau sollte bei Rot „in die Schule gehen, so, wie Rot bei Blau in die Schule gegangen ist. Zweitens, Blau sollte ein allgemein gehaltenes Gegenmodell zur roten Theorie des Sieges entwickeln. Drittens, Blau sollte dieses Modell auf spezifische regionale Kontexte zuschneiden. Die Theorie muss das gesamte Konfliktspektrum abdecken: von Konflikten in der Grauzone über Krisen und Kriege bis zur Beendigung eines Krieges. Ihr Kernkonzept sollte nicht Abschreckung oder Eskalationskontrolle sein; vielmehr sollte sie sich darauf konzentrieren, das Vertrauen der Führungsspitzen in Russland und China in ihr Eskalationskalkül zu erschüttern. Blau muss in der Lage sein, den erwarteten Nutzen von Handlungen, die mit der roten Theorie des Sieges in Einklang stehen, zu verringern und gleichzeitig die von Rot erwarteten Kosten und Risiken in die Höhe zu treiben. Dies ist eine Gegeneskalationsstrategie, keine Eskalationsdominanzstrategie. Die blaue Theorie des Sieges muss auch berücksichtigen, dass es zwei unterschiedliche
Operationsgebiete gibt (Europa und Ostasien), aus denen in Krisen- und Kriegszeiten möglicherweise Ressourcen abgezogen werden. Eine glaubhafte Theorie des Sieges in dem dann vernachlässigten zweiten Operationsgebiet verlangt, dass sich die Vereinigten Staaten verstärkt auf Abschreckungsfähigkeiten ihrer Alliierten stützen und eine größere Bereitschaft zeigen, ein glaubhaftes nukleares Abschreckungsmittel für dieses spezifische Problem bereitzustellen.

Blaue Theorien des Sieges müssen auch Grauzonen-Konflikte berücksichtigen. Moskau und Peking sind offenkundig bestrebt, die regionalen Ordnungen in ihrem Umfeld Schritt für Schritt umzugestalten, aber immer in einer Weise, die unterhalb der Schwelle einer militärischen Antwort durch die Vereinigten Staaten und/ oder ihre Verbündeten bleibt. Blau verfügt hier allenfalls über eine rudimentäre Theorie des Sieges. Die Vereinigten Staaten und ihre Verbündeten können die bestehenden regionalen Sicherheitsordnungen aufrechterhalten, sie können Vorteile konsolidieren und diese Ordnungen selektiv erweitern und vertiefen, indem sie ihr Bekenntnis zur kollektiven Sicherheit, zu Freihandel und zu gemeinsamen Werten aufrechterhalten.

Die Entwicklung kohärenter regionaler Abschreckungsstrategien und der zugehörigen Siegestheorien für das gesamte Konfliktspektrum verlangt eine Erneuerung der Institutionen und der Expertise, die solche Strategiekonzepte erarbeiten können. Wenn sie diese intellektuellen Hausaufgaben nicht machen, werden die Vereinigten Staaten und ihre Verbündeten, auch wenn sie über schlagkräftige - militärische und anderweitige - Instrumente verfügen, keinen kohärenten theoretischen Bezugsrahmen besitzen, der aufzeigt, wie diese eingesetzt werden sollten. Im Falle eines Krieges „könnten wir verlieren,“ um die Worte der National Defense Strategy Commission zu benutzen. Oder wir könnten gewinnen - aber in einer Weise, die den Boden für Ressentiments und weitere Konflikte bereitet. Ohne eine solche Theorie könnten die Führungen in Moskau und Peking dazu ermutigt werden, Krisen herbeizuführen, und die Regierungen in verbündeten Ländern könnten sich für Unabhängigkeit und den Erwerb von Massenvernichtungswaffen entscheiden. Ohne eine solche Theorie werden sich die Schwächung der Machtstellung Amerikas, die die National Defense Strategy Commission 2018 beschrieb, und mit ihr die Krise der liberalen Weltordnung nur weiter verschlimmern. Die Erneuerung robuster Abschreckungsarchitekturen in Europa und Nordostasien ist der beste Weg, um sicherzustellen, dass die Führungen in Moskau und Peking das Interesse daran verlieren, die regionalen Ordnungen in ihrem Umfeld zu zerstören. In diesem Fall wird die Abschreckung ihren 
Zweck erfüllt haben, indem sie die Voraussetzungen für eine Verringerung der internationalen Spannungen schuf. Kurzum, eine Theorie des Sieges ist eine notwendige Bedingung für strategische Kompetenz und strategischen Erfolg.

\section{Literatur}

Adamsky, Dmitry (2019): Russian Nuclear Orthodoxy. Stanford, Calif.: Stanford University Press

Brauss, Heinrich (2018): NATO Beyond 70: Renewing a Culture of Readiness. Tallinn: International Centre for Defence and Security

Carr, Edward Hallet (1939): The Twenty Years Crisis: 1919-1939. London: MacMillan Press

Edelman, Eric/Roughead, Gary/Fox, Christine/Hicks, Kathleen/ Keane, Jack/Krepinevich, Andrew/Kyl, Jon/Mahnken, Thomas/ McCord, Michael/Morell, Michael/Patterson, Anne/Zakheim, Roger (2018): Providing for the Common Defense: The Assessment and Recommendations of the National Defense Strategy Commission. Washington, D.C.: US Institute of Peace

Erickson, Andrew S./Martinson, Ryan D., eds. (2019): China's Maritime Gray Zone Operations. Annapolis, Md.: Naval Institute Press

Fitzsimmons, Michael (2017): The False Allure of Escalation Dominance, War on the Rocks (November 16, 2017); https:// warontherocks.com/2017/11/false-allure-escalationdominance/

Hoffman, Frank G. (2019): Examining Complex Forms of Conflict: Gray Zone and Hybrid Challenges, PRISM 7, (4), 36

Hoffman, Frank G. (2020): The Missing Element in Crafting National Strategy: a Theory of Success, Joint Forces Quarterly, 97 (2), 55-64

Jonsson, Oscar (2019): The Russian Understanding of War: Blurring the Lines Between War and Peace. Washington, DC: Georgetown University Press

Kahn, Herman (1965): On Escalation: Metaphors and Scenarios. London: Routledge

Krause, Joachim (2020): Kriege und Kriegsgefahren im kommenden Jahrzehnt, Sirius -Zeitschrift für strategische Analysen, 4 (2), 117-143

Kulesa, Lukasz (2018): Envisioning a Russia-NATO Conflict: Implications for Deterrence Stability: A Euro-Atlantic Security Report. London: European Leadership Network
Lindsay, Jon R./Gartzke, Erik, eds. (2019): Cross-Domain Deterrence: Strategy in an Era of Complexity. Oxford: Oxford University Press

Mitre, Jim (2019): A Eulogy for the Two-War Construct, Washington Quarterly, 41 (4), 7-30

Mulvenon, James/Finkelstein, David, eds. (2005): China's Revolution in Doctrinal Affairs: Emerging Trends in the Operational Art of the Chinese People's Liberation Army. Alexandria, VA: CNA Corporation

Nitze, Paul (1956): Atoms, Strategy, and Policy, Foreign Affairs, 35 (2), 187-198

Peng, Guangqian/Yao, Youzhi, eds. (2015): The Science of Military Strategy. Beijing: PLA

Qiao, Liang/Wang, Xiangsui (1999): Unrestricted Warfare. Beijing: PLA

Roberts, Brad (2013): Extended Deterrence and Strategic Stability in Northeast Asia. Tokyo: National Institute for Defense Studies

Roberts, Brad (2015): The Case for U.S. Nuclear Weapons in the 21 Century. Stanford, Calif.: Stanford University Press

Roberts, Brad (2019): On Creating the Conditions for Nuclear Disarmament: Past Lessons, Future Prospects, Washington Quarterly, 42 (2), 7-30

Roberts, Peter (2017): Designing Conceptual Failure in Warfare, RUSI Journal, 162 (1), 14-23

Schelling, Thomas (1960a): Strategy of Conflict. Cambridge, Mass.: Harvard College

Schelling, Thomas C. (1960b): Foreword, in: Roberta Wohlstetter: Pearl Harbor: Warning and Decision. Stanford, Calif.: Stanford University Press

Scobell, Andrew/Kamphausen, Roy D./Lai, David, eds. (2009): PLA Missions Beyond Taiwan. Washington, D.C.: Strategic Studies Institute of the U.S. Army War College and National Bureau of Asian Research

Swaine, Michael D./Zhang, Tuosheng/Cohen, Danielle (2006): Managing Sino-American Crises: Case Studies and Analysis. Washington, DC: Carnegie Endowment for International Peace

Talmadge, Caitlin (2019): Emerging Technology and Intra-War Escalation Risks: Evidence from the Cold War, Implications for Today, Journal of Strategic Studies, 42 (6), 864-887

Thomas, Timothy L. (2015): Russian Military Strategy: Impacting 21st Century Reform and Geopolitics. Ft. Leavenworth, Tx.: Foreign Military Studies Office

Thornhill, Paula/Karlin, Mara (2018): The Chairman the Pentagon Needs, War on the Rocks, January 5, 2018; https://waronthe rocks.com/2018/01/chairman-pentagon-needs/ 